\title{
Effectiveness of high-throughput miniaturized sorbent- and solid phase microextraction techniques combined with gas chromatography-mass spectrometry analysis for a rapid screening of volatile and semi-volatile composition of wines-A comparative study
}

\author{
Berta Mendes, João Gonçalves, José S. Câmara* \\ CQM - Centro de Química da Madeira, Centro de Ciências Exactas e da Engenharia da Universidade da Madeira, Campus Universitário da Penteada, 9000-390 Funchal, Portugal
}

\section{A R T I C L E I N F O}

\section{Article history:}

Received 8 June 2011

Received in revised form

28 September 2011

Accepted 12 October 2011

Available online 17 October 2011

\section{Keywords:}

Wines

Global fingerprint

VOCs and SVOCs

Solid phase extraction

Microextraction by packed sorbent

Solid phase microextraction

\begin{abstract}
A B S T R A C T
In this study the feasibility of different extraction procedures was evaluated in order to test their potential for the extraction of the volatile (VOCs) and semi-volatile constituents (SVOCs) from wines. In this sense, and before they could be analysed by gas chromatography-quadrupole first stage masss spectrometry (GC-qMS), three different high-throughput miniaturized (ad)sorptive extraction techniques, based on solid phase extraction (SPE), microextraction by packed sorbents (MEPS) and solid phase microextraction (SPME), were studied for the first time together, for the extraction step. To achieve the most complete volatile and semi-volatile signature, distinct SPE (LiChrolut EN, Poropak Q Styrene-Divinylbenzene and Amberlite XAD-2) and MEPS $\left(C_{2}, C_{8}, C_{18}\right.$, Silica and M1 (mixed $\left.C_{8}-S C X\right)$ ) sorbent materials, and different SPME fibre coatings (PA, PDMS, PEG, DVB/CAR/PDMS, PDMS/DVB, and CAR/PDMS), were tested and compared. All the extraction techniques were followed by GC-qMS analysis, which allowed the identification of up to 103 VOCs and SVOCs, distributed by distinct chemical families: higher alcohols, esters, fatty acids, carbonyl compounds and furan compounds. Mass spectra, standard compounds and retention index were used for identification purposes.

SPE technique, using LiChrolut $\mathrm{EN}$ as sorbent $\left(\mathrm{SPE}_{\mathrm{LiChrolut}} \mathrm{EN}\right.$ ), was the most efficient method allowing for the identification of 78 VOCs and SVOCs, 63 and 19 more than MEPS and SPME techniques, respectively. In MEPS technique the best results in terms of number of extractable/identified compounds and total peak areas of volatile and semi-volatile fraction, were obtained by using $C_{8}$ resin whereas DVB/CAR/PDMS was revealed the most efficient SPME coating to extract VOCs and SVOCs from Bual wine. Diethyl malate $(18.8 \pm 3.2 \%)$ was the main component found in wine $\mathrm{SPE}_{\mathrm{LiChrolut} E \mathrm{~N}}$ extracts followed by ethyl succinate (13.5 $\pm 5.3 \%)$, 3-methyl-1-butanol (13.2 $\pm 1.7 \%)$, and 2-phenylethanol (11.2 $\pm 9.9 \%)$, while in $\mathrm{SPME}_{\mathrm{DVB} / \mathrm{CAR} / \mathrm{PDMS}}$ technique 3-methyl-1-butanol (43.3 $\left.\pm 0.6 \%\right)$ followed by diethyl succinate $(18.9 \pm 1.6 \%)$, and 2 -furfural ( $10.4 \pm 0.4 \%$ ), are the major compounds. The major VOCs and SVOCs isolated by MEPS ${ }_{\mathrm{C} 8}$ were 3-methyl-1-butanol ( $26.8 \pm 0.6 \%$, from wine total volatile fraction), diethyl succinate $(24.9 \pm 0.8 \%)$, and diethyl malate $(16.3 \pm 0.9 \%)$. Regardless of the extraction technique, the highest extraction efficiency corresponds to esters and higher alcohols and the lowest to fatty acids.

Despite some drawbacks associated with the SPE procedure such as the use of organic solvents, the time-consuming and tedious sampling procedure, it was observed that $\mathrm{SPE}_{\mathrm{LiChrolut}} \mathrm{EN}$, revealed to be the most effective technique allowing the extraction of a higher number of compounds (78) rather than the other extraction techniques studied.
\end{abstract}

(c) 2011 Elsevier B.V. All rights reserved.

\section{Introduction}

The volatile fraction of wine plays a prominent role in its organoleptic characteristics. It determines their aroma, which is the major contributor to overall flavor perception and one

\footnotetext{
* Corresponding author.: Tel.: +351 291 705112; fax: +351 291705149.

E-mail address: jsc@uma.pt (J.S. Câmara).
}

of the most important parameters influencing the wine quality and consumer acceptance [1,2]. Their chemical composition contains numerous small molecules (over 1000 volatile compounds) belonging to distinct chemical families, including monoterpenoids, $\mathrm{C}_{13}$-norisoprenoids, sesquiterpenoids, higher alcohols, ethyl esters, fatty acids, acetates, isoamyl esters, carbonyls, sulphurs, furan compounds, and volatile phenols. Moreover, these compounds have different physicochemical properties regarding concentration (ranging from several $\mathrm{mgL}^{-1}$ (e.g., ethyl acetate) to less than a 
Table 1

Basics of the considered extraction techniques.

\begin{tabular}{|c|c|c|c|c|}
\hline \multirow[t]{2}{*}{ Sorbent materials } & \multicolumn{4}{|l|}{ SPE } \\
\hline & Type & Interactions & Coating stability & Retention mechanism \\
\hline LiChrolut EN (40-120 $\mu \mathrm{m})$ & Porous polymer & Non polar & Hypercrosslinked & Sorption and partition \\
\hline Porapak Q(50-80 mesh) & Porous polymer & Non polar & Partially crosslinked & Sorption and partition \\
\hline Styrene-DVB (18-100 mesh) & Macroporous polymer & Polar & Partially crosslinked & Sorption and partition \\
\hline Amberlite XAD-2 (20-60 mesh) & Porous polymer & Polar & Partially crosslinked & Sorption and partition \\
\hline \multirow[t]{2}{*}{ Sorbent materials } & \multicolumn{4}{|l|}{ MEPS } \\
\hline & Type & Interactions & Coating stability & Retention mechanism \\
\hline Octadecyl $\left(\mathrm{C}_{18}\right)^{\mathrm{a}, \mathrm{b}}$ & Polymer & Non polar & Partially crosslinked & Sorption and partition \\
\hline Octyl $\left(C_{8}\right)^{a, c}$ & Polymer & Non polar & Partially crosslinked & Sorption and partition \\
\hline Ethyl $\left(C_{2}\right)^{a, d}$ & Polymer & Non polar & Partially crosslinked & Sorption and partition \\
\hline $\operatorname{Mixed}\left(\mathrm{C}_{8}-\mathrm{SCX}\right) \mathrm{M}_{1}^{\mathrm{a}, \mathrm{e}, \mathrm{f}}$ & Polymer & Bipolar & Partially crosslinked & Sorption and partition \\
\hline Silica (SIL) $)^{\mathrm{a}, \mathrm{f}}$ & Porous particle & Polar & Partially crosslinked & Sorption and partition \\
\hline \multirow[t]{2}{*}{ Coating ${ }^{g}$} & \multicolumn{4}{|l|}{ SPME } \\
\hline & Type & Interactions & Coating stability & Retention mechanism \\
\hline PDMS $(100 \mu \mathrm{m})$ & Homogeneous polymer & Nonpolar & Nonbonded & Absorption \\
\hline PDMS/DVB $(65 \mu \mathrm{m})$ & Porous particle/polymer & Bipolar & Partially crosslinked & Adsorption \\
\hline $\mathrm{PA}(85 \mu \mathrm{m})$ & Homogeneous polymer & Polar & Bonded crosslinked & Absorption \\
\hline PEG $(60 \mu \mathrm{m})$ & Homogeneous polymer & Polar & Highly crosslinked & Absorption \\
\hline CAR/PDMS (75 $\mu \mathrm{m})$ & Porous particle/polymer & Bipolar & Partially crosslinked & Adsorption \\
\hline DVB/CAR/PDMS $(50 / 30 \mu \mathrm{m})$ & Porous particle/polymer & Polar & Highly crosslinked & Adsorption \\
\hline
\end{tabular}

a The base material is silica with mean particle and pore size of $50 \mu \mathrm{m}$ and $60 \AA$, respectively.

b Low carbon load $\mathrm{C}_{18}$; general purpose phase.

${ }^{c}$ Less hydrophobic than $C_{18}$; less retention of highly hydrophobic compounds; used when $C_{18}$ is to retentive.

d Short chain functional group is less hydrophobic than $\mathrm{C}_{8}$; less retention of hydrophobic compounds; used when $\mathrm{C}_{8}$ is to retentive.

e Mixed-mode sorbents constituted by $\mathrm{C}_{8}$ and SCX copolymer.

f Highly polar surface; most common polar phase.

g PDMS, polydimethylsiloxane; PDMS/DVB, polydimethylsiloxane/divinylbenzene; PA, polyacrylate; PEG, polyethylene glycol; CAR/PDMS, CARboxen $^{\mathrm{TM}} /$ Polydimethylsiloxane; DVB/CAR/PDMS, divinylbenzene/carboxen/polydimethylsiloxane.

few ng $\mathrm{L}^{-1}$ (e.g., 3-isobutyl-2-methoxypyrazine, IBMP)), polarity, volatility and odour impact [3,4]. They are largely derived from four different sources: (i) the grape berry; (ii) processing of the grapes (namely crushing, pressing, etc.) by chemical, enzymatic-chemical and thermal reaction in grape must; (iii) the yeast strain used for fermentation; (iv) from containers used for wine making (wood, commonly oak) and chemical reactions during maturation wine storage [1].

Owing to the complex nature of the wine matrix, there is a consensus on that efficient sample preparation, trace-level detection and identification are important aspects of analytical methods to determine VOCs and SVOCs in wines. Many of the reported methods relies on extraction with organic solvents, including conventional techniques such as liquid-liquid extraction (LLE) [5], static or dynamic headspace extraction (SHS, DHS) [6], supercritical fluid extraction (SFE) [7], soxhlet extraction [8], and ultrasound extraction (USE) [9]. The broad polarity range of solvents and its general applicability made these techniques very popular [10]. However, most of these approaches present important drawbacks, typically are time-consuming and labour-intensive, uses of significant amounts of environmentally unfriendly solvents, and involves multi-step procedures, which can lead to analyte losses and a reduction in sensitivity. Finally, but also of importance, is the fact that many aroma compounds are chemically very unstable and can be easily oxidized or thermo degraded [4]. So, the search and development of adequate extraction techniques, that minimize the use of harmful organic solvents and/or even solvent-free procedures, and therefore more sustainable and easily implemented, has attracted the attention of many scientists. Therefore, in recent years miniaturized analytical techniques $[11,12]$ had gained attention due to its many special features over conventional approaches [5-8]. Among many advantages, usage of little or no solvent, the low volumes of sample required, the greater sensitivity in sample preparation than do the exhaustive extraction procedures, increasing of sensitivity of analysis and user-friendly system, should be pointed out (Table 1). So, relatively new miniaturized extraction techniques, such as microextraction by packed sorbents (MEPS), solid phase microextraction (SPME), and stir bar sorptive extraction (SBSE), have been gradually replacing conventional analytical methods.

The technique most frequently used for wine VOCs and SVOCs extraction or clean-up is SPE, which involves a liquid-solid partitioning, and the analytes are bound to active sites on the surface of solid sorbent. The possibility of using different sorbents for trapping analytes over a wide range of polarities, such as highly cross-linked copolymers, functionalized copolymers, graphitized carbons or some specific $n$-alkyl silicas, and eluents makes SPE a very selective technique (Table 1 ). The fact that only minor amounts of organic solvents are used compared to LLE, is why SPE has been extensively used for the analysis of volatile aroma compounds $[8,13-16]$ and off-flavors $[17,18]$ in wines.

More recently the conventional SPE ( $\mathrm{mL}$ bed volumes) has been adopted for microextraction through a syringe packed with suitable solid phase material, a novel method for sample preparation and sample handling - microextraction by packed sorbent (MEPS) ( $\mu \mathrm{L}$ volumes). Is a miniaturization of the conventional SPE in which the sample volume and volumes for extraction and washing solvents are reduced compared to SPE technique [19] (Table 2). A wide range of sorbents are available including $C_{2}, C_{8}$ and $C_{18}$ bonded phases on silica, polymeric resins (polystyrene/divinylbenzene copolymer), molecular imprinted polymers (MIPs), polar sorbents such as silica and ion-exchange sorbents, and mixed-mode sorbents. These different phases enable interactions based on adsorption, H-bonding, polar and nonpolar interactions, cation, anion exchange or size exclusion. This new technique is very promising because it is fast, simple, requires very small volume of samples (few $\mu \mathrm{L}$ ) to produce comparable results to conventional SPE technique, and the cost of 
Table 2

Schematic diagrams and comparison of a range of factors of the extraction techniques used in the study for liquid samples.

\begin{tabular}{|c|c|c|}
\hline SPE & MEPS & SPME \\
\hline Automation available & Automation possible & Automation possible \\
\hline Difficult to put on line & Designed for on-line (GC-MS, LC-MS) & Designed for on-line (GC-MS, LC-MS) \\
\hline No emulsions & No emulsions & No emulsions \\
\hline Parallel operation gives high throughput & Low volumes gives a fast method & Fast and reproducible method \\
\hline Polar and charged analytes may be extracted & Polar and charged analytes may be extracted & Charged analytes are normally not efficiently extracted \\
\hline High sample size and moderate solvent volume & Low sample size and solvent volume & Solvent-free \\
\hline $\begin{array}{l}\text { Depleted sample may be recovered } \\
\text { uncontaminated }\end{array}$ & Depleted sample may be recovered uncontaminated & Depleted sample may be recovered uncontaminated \\
\hline $15-30 \mathrm{~min}$ of extraction time & $2-10 \min$ & $15-60 \mathrm{~min}$ \\
\hline $\begin{array}{l}\text { Semi-volatile to non-volatile, nonpolar to polar, } \\
\text { and ionisable analytes }\end{array}$ & $\begin{array}{l}\text { Semi-volatile to non-volatile, nonpolar to polar, and } \\
\text { ionisable analytes }\end{array}$ & $\begin{array}{l}\text { Suited for very volatile to semi-volatile, nonpolar and polar } \\
\text { analytes }\end{array}$ \\
\hline \multirow[t]{2}{*}{ Excellent repeatability } & Good repeatability & Good repeatability \\
\hline & Suitable for on-site sampling & Suitable for on-site and in vivo sampling \\
\hline Few extractions & $80-100^{\mathrm{a}}$ extractions & Fibre used in $80-100^{\text {a }}$ extractions \\
\hline Quantitative extraction & Quantitative extraction & Not quantitative extraction \\
\hline $\begin{array}{l}\text { A large range of sorbents are available, from } \\
\text { nonpolar to ion-exchange materials }\end{array}$ & $\begin{array}{l}\text { A large range of sorbents are available, from nonpolar } \\
\text { to ion-exchange materials }\end{array}$ & Limited range of stationary phases \\
\hline Off-line & $\begin{array}{l}\text { Can be used off-line or connected on-line with LC, GC, } \\
\text { LC-MS, GC-MS and CE }\end{array}$ & $\begin{array}{l}\text { Can be used off-line or connected on-line with LC, GC, } \\
\text { LC-MS, GC-MS and CE }\end{array}$ \\
\hline
\end{tabular}

a Depends on the nature and purity of the matrix. According to Nerín et al. [19] the packed syringe can be used more than 400 times for water sample.

analysis is minimal compared to SPE (Table 2). Furthermore, this technique can be easily interfaced to LC-MS and GC-MS to provide a completely automated MEPS/LC-MS or MEPS/GC-MS system [20].

The MEPS technique has been used to extract various analytes from biological samples. Several drugs such as local anaesthetics and their metabolites [21]; the anticancer drugs roscovitine [22], olomoucine [23], and busulfan [24]; the $\beta$-blocker drugs acebutolol and metoprolol [25]; the anti-depressant drugs dopamine and serotonine [26] as well as anti-addictive methadone [27] have been successfully extracted by MEPS from biological samples such as plasma, urine or blood.

Solid-phase microextraction (SPME) emerged as a versatile alternative method of analyte extraction and pre-concentration, which requires little or no organic solvents, is easily automated, and can also improve the limits of detection [28]. SPME encompasses sampling, extraction, pre-concentration and introduction of the sample into the system of analyses in a single uninterrupted process, thus avoiding contamination of the matrix [28]. Generally accepted drawbacks are a relatively poor reproducibility, lot-to-lot variations, lack of selectivity, sensitivity against organic solvents, and the limited range of stationary phases which are commercially available, only roughly covering the scale of polarity [28]. Fibres are available coated with pure liquid polymeric phases or with porous solid phases (more accurately, porous solids dispersed in liquid polymer matrixes), in different coating combinations (Table 1 ), blends or copolymers, film thickness, and fibre assemblies enlarging to a certain extend the field of possible applications. The theoretical foundations of SPME have been extensively addressed in the literature [29-32]. Since its development, this technique has become very popular and gained growing acceptance and increasing use in routine laboratories applications mainly to the sampling and analysis of environmental [33], food [34-36], forensic [37] and pharmaceutical samples [38,39]. Typically followed by GC in combination with mass spectrometric detection (MS) [40-42] or its multidimensional alternative, comprehensive two-dimensional GC (GC $\times$ GC-ToFMS) [43-45], SPME technique has been successfully used for wine samples.
Based on the volatile and semi-volatile global composition of Bual wines, we aimed to evaluate and compare the extraction potential of three different miniaturized (ad)sorptive extraction techniques, based on solid phase extraction (SPE), microextraction in packed syringe (MEPS), and solid phase microextraction (SPME), followed by gas chromatography-mass spectrometry (GC-qMS). A comparative study of several SPE sorbent materials (the polymeric sorbents LiChrolut EN, Poropak $\mathrm{Q}$, styrene-divinylbenzene (Sty-DVB) and Amberlite XAD-2), different MEPS adsorbents (the classical bonded hydrocarbon phase RP- $C_{18},-C_{8},-C_{2}$, silica (SIL) and mixed-mode $\mathrm{C}_{8}$-SCX sorbent: M1), using dichloromethane (DCM) as solvent, and all commercially available SPME fibre coatings (PA, PDMS, PEG, DVB/CAR/PDMS, PDMS/DVB, and CAR/PDMS), were evaluated, in order to obtain an in-depth characterization of the volatile and semi-volatile signature of Bual wine, and to select the best sorbent/fibre for further investigations.

Although many studies reporting the comparison between different extraction methodologies for the analysis of wine VOCs and SVOCs compounds have been published, as far we know, this is the first work aimed to test the effectiveness of miniaturized (ad)sorptive extraction techniques, SPE, MEPS and SPME, for the first time together. Moreover, MEPS applied to the analysis of wine volatile and semi-volatile constituents has been poorly studied [46]. The study presented herein represents a first approach of the analysis of wine volatile profile using MEPS.

\section{Experimental}

\subsection{Reagents and materials}

All chemicals were of analytical grade and were used without further purification. Ethanol absolute (EtOH) (99.5\%), sodium chloride $(\mathrm{NaCl})$ (AR Grade) and sodium sulphate anhydrous $\left(\mathrm{Na}_{2} \mathrm{SO}_{4}\right)$ (Panreac, Barcelone, Spain). were obtained from Labodidáctica Equipamentos de Laboratório e Didácticos, Lda (Funchal, Portugal). Dichloromethane (DCM) (99.99\%) and methanol (MeOH) (HPLC gradient grade) were purchased from Fisher Scientific (Leicestershire, UK), ethyl acetate (99.8\%) and diethyl ether (99.5\%) from LAB 
SCAN analytical sciences (Gliwice, Poland) and octan-3-ol (99\%) from Sigma-Aldrich (Madrid, Spain). Ultra-pure water was prepared using a Milli-Q Plus water purification system to a resistivity of $18 \mathrm{M} \Omega \mathrm{cm}$ (Millipore Corporation (Bedford, MA, USA). Ultra-high purity (UHP) gases for chromatography were obtained from Air Liquide (Portugal).

The SPE sorbents, Porapak Q 50/80 mesh, Dow Styrene-DVB 18100 and Amberlite XAD-2 (Sigma) and Lichrolut EN 40-120 $\mu \mathrm{m}$ (Merck, Darmstadt, Germany). A Visiprep ${ }^{\mathrm{TM}}$ SPE vacuum manifold (12-port model, Supelco), the manifold accessories, and the KNF-Laboport ${ }^{\circledR}$ vacuum pump for vacuum manifold were from Sigma-Aldrich (Bellefonte, PA, USA). Solid phases used in MEPS procedure $\left(\mathrm{C}_{2}, \mathrm{C}_{8}, \mathrm{C}_{18}\right.$, SIL, and M1 (mix-mode $\mathrm{C}_{8}$-SCX)), the MEPS BIN and syringes, from SGE Analytical Science (SGE Europe Ltd., United Kingdom), were purchased from SGE Analytical Science (SGE Europe Ltd., United Kingdom).

The SPME fibres (Supelco, Bellefonte, PA, USA) coated with the following polymers: polydimethylsiloxane (PDMS, $100 \mu \mathrm{m}$ ), polyacrylate (PA, $85 \mu \mathrm{m}$ ), divinylbenzene/carboxen on polydimethylsiloxane (DBV/CAR/PDMS; StableFlex, 50/30 $\mu \mathrm{m}$ ), carboxen/polydimethylsiloxane (CAR/PDMS, $75 \mu \mathrm{m}$ ) and polydimethylsiloxane/divinylbenzene (PDMS/DVB, $65 \mu \mathrm{m}$ ), and poliethyleneglicol (PEG, $60 \mu \mathrm{m}$ ), the SPME holder for manual sampling, clear glass screw cap vials for SPME with PTFE/silica (film thickness $1.3 \mathrm{~mm}$ ) septa from Supelco (Bellefonte, PA, USA). Prior to initial use, all new fibres were conditioned as per the manufacturer's recommendations by heating in the injection port of the GC. Before the initial application, blank runs were completed before each sampling to ensure no carry-over of analytes from the previous extraction.

\subsection{Wine samples}

A monovarietal Madeira wine (medium sweet) with 5 years old (vintage 2005) with alcoholic degree of $16.0 \%$ (v/v) produced from Vitis vinifera $\mathrm{L}$. Bual grape varieties, and a $\mathrm{pH}$ value of 3.4 , was used in this study. The samples were kindly provided by Madeira Wine Company (MWC), Madeira Island, Portugal. All samples were kept at $-20^{\circ} \mathrm{C}$ until analysis. All analysis were carried out in triplicate.

\subsection{Extraction procedures}

Three different miniaturized extraction techniques, the conventional SPE, and the recent MEPS (using different sorbents and DCM as extracting solvent), and SPME in headspace mode, were tested and compared in order to evaluate their ability to extract VOCs and SVOCs from wines. Each technique has its own advantages and disadvantages, some of which are summarised in Table 2 . To select a suitable sample pre-treatment technique, several factors such as the nature of analytes, the type of sample, the simplicity and ruggedness of the technique, the time required for the sample treatment, and the subsequent analytical technique are the key factors [10].

\subsubsection{Solid phase extraction (SPE)}

Four distinct hydrophilic reversed-phase SPE sorbents: LiChrolut EN (styrene-divinylbenzene copolymer), Porapak Q (ethylvinylbenzene-divinylbenzene copolymer), styrene-divinylbenzene copolymer (Sty-DVB), and Amberlite XAD-2 (styrene-divinylbenzene copolymer), were evaluated and compared. Styrene-DVB resin has been poorly studied, as opposed to other sorbents $[47,48]$.

Each cartridge, containing $200 \mathrm{mg}$ of sorbent, was processed manually with a SPE Visiprep ${ }^{\mathrm{TM}} 12$-port vacuum manifold (Sigma-Aldrich). LiChrolut EN resin was activated with $4 \mathrm{~mL}$ of $\mathrm{DCM}, 2 \mathrm{~mL}$ of $\mathrm{MeOH}$ and, finally with $4 \mathrm{~mL}$ water-EtOH solution
(13\%, v/v). For Sty-DVB and Porapak Q sorbents, the conditioning was carried out using $4 \mathrm{~mL}$ of $\mathrm{MeOH}$ and $2 \mathrm{~mL}$ of water. Amberlite XAD-2 resin, previously washed according to Edwards and Beelman procedure [49] to remove impurities, and conditioned with $2 \mathrm{M}$ of $\mathrm{HCl}$ in $\mathrm{MeOH}$. The extractions were performed according to the methodology proposed by Zapata et al. [50]. Briefly, $5 \mathrm{~mL}$ of wine, containing $200 \mu \mathrm{L}$ of internal standard (octan-3-ol, $3 \mathrm{mg} \mathrm{L}^{-1}$ ), were passed through the SPE cartridge at around $1.5 \mathrm{~mL} \mathrm{~min}^{-1}$. After this, the sorbent was dried by letting air pass through it. The adsorbents were washed with $4 \mathrm{~mL}$ of Milli-Q ultra-pure water to remove interferents. The analytes were recovered by elution with $1 \mathrm{~mL}$ of DCM. The organic extracts were dehydrated with anhydrous sodium sulphate and concentrated under $\mathrm{N}_{2}$ atmosphere to a volume of $200 \mu \mathrm{L}$. Then $1 \mu \mathrm{L}$ of extract were injected in GC-qMS system. The analyses were performed in triplicate.

\subsubsection{Microextraction by packed sorbent (MEPS)}

Using the MEPS procedure, the sample pre-treatment takes place on the packed bed, available in a variety of sorbents and inserted inside a syringe between the barrel and the needle (Barrel Insert and Needle Assembly, BIN). The MEPS BIN is easily installed into the syringe housing and then secured by a locking nut.

In the present work, MEPS was carried out using different BINs each containing $1 \mathrm{mg}$ of based solid-phase material silica- $\mathrm{C}_{2}, \mathrm{C}_{8}$, $\mathrm{C}_{18}$, SIL and M1 (mixed-mode $\mathrm{C}_{8}+$ SCX sorbent; particle size $50 \mu \mathrm{m}$ ) inserted into a $250 \mu \mathrm{L}$ gas-tight syringe. Before being used for the first time, the sorbents were manually conditioned firstly with $50 \mu \mathrm{L}$ of $\mathrm{MeOH}$ and then with $50 \mu \mathrm{L}$ of $0.1 \%$ formic acid solution. After that, an aliquot of $100 \mu \mathrm{L}$ of sample was drawn through the syringe up and down 10 times (multiple extraction cycles, increases the extraction recovery) from different aliquots (extract-discard). The sorbent was washed once with $100 \mu \mathrm{L}$ of $0.1 \%$ formic acid to remove interferences. The analytes were then eluted with $250 \mu \mathrm{L}$ DCM directly into the autosampler vials. After extraction, and in order to minimize/eliminate carry-over, the stationary phase was washed with $250 \mu \mathrm{L}$ of $\mathrm{MeOH}$ and $250 \mu \mathrm{L} 0.1 \%$ formic acid solution. To reuse MEPS cartridge the sorbent was washed 3-4 times with water and 3-4 with solvent (elution solution), to remove unwanted weakly retained interferences.

\subsubsection{Solid phase microextraction (SPME)}

To test the potentialities of SPME extraction technique and to compared them with the SPE and MEPS techniques, extractions were performed in the headspace mode using six distinct SPME fibres commercially available, namely a tripolar phase (divinylbenzene/carboxen/polydimentylsiloxane, DVB/CAR/PDMS 50/30 $\mu \mathrm{m}$ ), two bipolar phases (carboxen/polydimentylsiloxane, CAR/PDMS $75 \mu \mathrm{m}$; and polydimentylsiloxane/divinylbenzene, PDMS/DVB $65 \mu \mathrm{m}$ ); two polar phases (polyethylene glycol, PEG $60 \mu \mathrm{m}$, and polyacrylate PA $85 \mu \mathrm{m}$ ); and a non-polar phase (polydimentylsiloxane, PDMS $100 \mu \mathrm{m}$ ). The fibres were conditioned before use as recommended by the manufacturer. Before the first daily analysis, the fibres were conditioned for $10 \mathrm{~min}$ at $250^{\circ} \mathrm{C}$ in the GC injection port to eliminate any possible carryover on the coated fibre.

The HS-SPME experimental parameters were previously established by Câmara et al. [2]. Briefly, aliquots of $4 \mathrm{~mL}$ of Bual wine were placed into 8-mL glass vial, which correspond to a ratio of the volume of the liquid phase to the headspace volume $(1 / \beta)$ of 0.5 . After the addition of $0.5 \mathrm{~g}$ of $\mathrm{NaCl}$ (added in order to improve the extraction efficiency by decreasing the solubility of hydrophilic compounds in the aqueous phase) and a micro stirring bar $(0.5 \mathrm{~mm} \times 0.1 \mathrm{~mm}$; Supelco $)$, the vial was capped with a PTFE septum and an aluminium cap (Chromacol, Hertfordshire, UK) and placed in a thermostat bath. All samples were incubated at $40.0 \pm 0.1^{\circ} \mathrm{C}$ with agitation at $800 \mathrm{rpm}$ for $5 \mathrm{~min}$ prior to extraction. Then the SPME fibre was manually inserted into the sample 
vial headspace for $60 \mathrm{~min}$ at $40.0 \pm 0.1^{\circ} \mathrm{C}$. As stirring of the solution usually improves the extraction, since the static layer resistant to mass transfer is destroyed (facilitate mass transport between the bulk of the aqueous sample and the fibre), all the experiments were performed under constant stirring velocity ( $800 \mathrm{rpm})$. Afterwards the SPME fibre was withdrawn into the needle, removed from the vial and inserted into the injection port of the GC-qMS system for 6 min where the analytes were thermally desorbed and transferred directly to the analytical column. Desorption temperatures, depending on the fibre used, ranging from $250^{\circ} \mathrm{C}$ for PDMS/DVB and PDMS to $300^{\circ} \mathrm{C}$ for CAR/PDMS. All measurements were performed in triplicate. Blanks, corresponding to the analysis of the coating fibre not submitted to any extraction procedure, were run between sets of five analyses.

\subsection{GC-qMS analysis}

The SPE and MEPS extracts and the SPME coating fibres containing VOCs and SVOCs of Bual wine were manually introduced into the GC injection port at $250^{\circ} \mathrm{C}$ (equipped with a glass liner, $0.75 \mathrm{~mm}$ i.d.). In SPME the fibres were kept for 5 min for VOCs and SVOCs thermal desorption. The desorbed VOCs and SVOCs were separated on a Agilent Technologies (Palo Alto, CA, USA) 6890N Network gas chromatograph equipped with a BP-20 (polyethylene glycol) fused silica capillary column $(30 \mathrm{~m} \times 0.25 \mathrm{~mm}$ i.d.; film thickness, $0.25 \mu \mathrm{m}$ ) from Scientific Glass Engineering (Milton Keynes, UK) and interfaced with an Agilent 5975 quadrupole inert mass selective detector. The injection volume for liquid extracts (SPE and MEPS) was $1 \mu \mathrm{L}$. Ultra-high purity (UHP) helium (Air Liquide, Portugal) was used as the carrier gas at a constant flow of $1.1 \mathrm{~mL} \mathrm{~min}^{-1}$ (column head pressure of $13 \mathrm{psi}$ ). For the analysis of the SPE and SPME extracts, the temperature program was $40^{\circ} \mathrm{C}$ for $3 \mathrm{~min}$, ramped at $1.7^{\circ} \mathrm{C} \mathrm{min}^{-1}$ to $200^{\circ} \mathrm{C}(3 \mathrm{~min})$ then to $220^{\circ} \mathrm{C}$ at $3^{\circ} \mathrm{C} \mathrm{min}^{-1}$ and held isothermally for a further $5 \mathrm{~min}$. For MEPS extracts, and being in consideration that the extracts are much more simple than the obtained by SPE and SPME techniques, it was used a GC oven temperature program slightly different with a shorter run time, $40^{\circ} \mathrm{C}$ $(1 \mathrm{~min})$ to $220^{\circ} \mathrm{C}(2 \mathrm{~min})$ ramped at $3^{\circ} \mathrm{C} \mathrm{min}^{-1}$, so the retention time of the analogues compounds is quite different. The rest of conditions were similar to the ones described.

The mass spectrometer was operated in the electron-impact(EI) ionisation mode at $70 \mathrm{eV}$. For the MS system the temperatures of the transfer line, quadrupole and ionisation source were 220, 180 and $230^{\circ} \mathrm{C}$, respectively. The ionisation was maintained off during the first 4 min to avoid solvent overloading with a source temperature of $230^{\circ} \mathrm{C}$. The mass spectra were recorded in range of 35-300 $\mathrm{m} / \mathrm{z}$ and the acquisition was made in Full Scan mode with a scan rate of 6 scans/s. The electron multiplier voltage was set in the relative mode to auto tune procedure. MS system was operated under identical conditions in all analysis (SPE, MEPS, and SPME).

Identification of VOCs and SVOCs was achieved (1) comparing the GC retention times and mass spectra, with those, when available, of the pure standard compounds, (2) all mass spectra were also compared with the data system library (NIST, 2005 software, Mass Spectral Search Program V.2.0d; NIST 2005, Washington, DC), and (3) Kovats retention index (RI) values calculated through injection of SPE $\mathrm{LiChrolut} \mathrm{EN}_{\text {and HS-SPME }}$ DVB/CAR/PDMS extracts of a series of $\mathrm{C}_{8}-\mathrm{C}_{20}$ straight-chain $n$-alkanes (concentration of $40 \mathrm{mg} \mathrm{L}^{-1}$ in $n$-hexane) according to the following expression:

$\mathrm{RI}(x)=100 \times z+100 \frac{\mathrm{RT}(x)-\mathrm{RT}(z)}{\mathrm{RT}(z+1)-\mathrm{RT}(z)}$

where $\operatorname{RI}(x)$ is the retention index of the unknown compound $x$, $z$ is the number of carbon atoms of the $n$-alkane eluted before the unknown compound $x, z+1$ is the number of carbon atoms of the $n$ alkane eluted after the unknown compound $x, \operatorname{RT}(x)$ is the retention time of the unknown compound $x, \operatorname{RT}(z)$ is the retention time of the $n$-alkane eluted before the unknown compound $x, \operatorname{RT}(z+1)$ is the $n$ alkane eluted after the unknown compound $x$. These values were compared, when available, with values reported in the literature for similar chromatographic columns [47].

The GC peak area data obtained were used as an indirect approach to estimate the relative content of each volatile and semivolatile compound.

\subsection{Statistical analysis}

Significant differences among the three extraction techniques were assessed with a one-way analysis of variance (ANOVA) using a SPSS Program, version 19.0 (SPSS Inc. Headquarters, Chicago IL, USA). The Least Square Difference (LSD) test ( $p$-value $<0.05$ ) was used to compare the means. Principal component analysis (PCA) was used in order to establish an objective comparison among the extraction techniques. PCA is a tool for data exploration which allows the reduction of the dimensionality of data facilitating the analysis of inter-technique relationships. New variables, so called principal components, are obtained to explain the greater part of total variance with a minimum of information loss. Figures and Tables were generated using Microsoft Office Excel 2007 (Microsoft Corporation, Redmond, WA, USA).

\section{Results and discussion}

Three extraction techniques, SPE, MEPS and SPME, were used to test their ability to extract VOCs and SVOCs from an aged (5 years old) medium sweet Madeira wine. The compounds are listed in Tables 3-5 following elution order, and including their chemical name, retention index for polar columns, the identification method used, and the corresponding odour descriptor. Differences in the chromatographic profiles were observed by using different sorbents. The identified compounds were organized in distinct chemical groups according to their chemical structure as higher alcohols (HA), esters (EST), volatile fatty acids (VFA), carbonyl compounds (CC) and furan compounds (FC), while compounds of differentiated structure were considered together in the class "miscellaneous" (others). The peak numbers of the chromatograms match the numbers of Tables 3-5.

\subsection{SPE analysis}

A comparison of different sorbents for the extraction of VOCs and SVOCs from Bual wines was carried by using hydrophobic sorbents. The sorbents selected were the classical polymeric sorbents (LiChrolut EN, Poropak Q; Sty-DVB, and Amberlite XAD-2). Fig. 1 shows a typical total ion chromatogram (TIC) of VOCs and SVOCs from a Bual wine obtained by SPE extraction technique with distinct sorbents.

Table 3 displays the compounds identified in the four SPE extracts derived from triplicate extractions, along with their retention indices (RI), odour description and peak area [51-61].

Effectiveness of the sorbents for wine volatile and semi-volatile constituents was evaluated by total peak area of the volatile fraction, reproducibility and number of the volatiles extracted. Among the four SPE sorbents evaluated, LiChrolut-EN clearly showed the highest extraction capacity as evidenced by previous studies $[8,62]$ suggesting that its retention ability for VOCs and SVOCs is much stronger than the remaining three sorbents. In this approach, and for comparison of the extraction capacity, the maximum analytical signal obtained from the LiChrolut EN extract was taken as $100 \%$, and the relative results for other SPE sorbent materials are shown in Fig. 2. 
Table 3

Volatile and semi-volatile constituents identified in Bual Madeira wine samples ( 5 years old) obtained by SPE followed by GC-qMS, the corresponding retention times, retention indices and odour descriptors.

\begin{tabular}{|c|c|c|c|c|c|c|c|c|c|c|}
\hline \multirow[t]{2}{*}{ Peak no. } & \multirow[t]{2}{*}{$\mathrm{RT}^{\mathrm{a}}(\min )$} & \multirow[t]{2}{*}{$\mathrm{RI}_{\mathrm{lit}}{ }^{\mathrm{b}}$} & \multirow[t]{2}{*}{$\mathrm{RI}_{\mathrm{cal}} \mathrm{c}$} & \multirow[t]{2}{*}{ Compound } & \multirow[t]{2}{*}{$\mathrm{ID}^{\mathrm{d}}$} & \multirow[t]{2}{*}{ Odour description } & \multicolumn{4}{|c|}{ SPE $\left[\right.$ Peak area $\left.\times 10^{6} \pm \operatorname{SD}(\%)\right](n=3)$} \\
\hline & & & & & & & LiChrolut EN & Poropak Q & Sty-DVBe & Amberlite XAD-2 \\
\hline 1 & 5.080 & 1046 & 1044 & 1-Propanol & $\mathrm{S}, \mathrm{MS}$ & Alcohol, pungent & $4.9 \pm 1.9$ & $0.8 \pm 6.7$ & n.d $d^{f}$ & n.d \\
\hline 2 & 5.352 & 1034 & 1049 & Ethyl butyrate & MS & Strawberry, fruity & $9.0 \pm 8.4$ & $1.5 \pm 6.6$ & n.d & n.d \\
\hline 3 & 5.773 & 1049 & 1057 & Ethyl 2-methylbutyrate & MS & Strawberry, berry & $1.6 \pm 8.3$ & n.d & n.d & n.d \\
\hline 4 & 5.966 & 1051 & 1061 & 1,1-Diethoxybutane & MS & Floral & $9.1 \pm 1.5$ & $0.9 \pm 4.5$ & n.d & n.d \\
\hline 5 & 6.267 & 1069 & 1066 & Ethyl 3-methylbutyrate & MS & Exotic fruits & $7.1 \pm 0.8$ & $1.8 \pm 2.8$ & n.d & n.d \\
\hline 6 & 6.796 & 1099 & 1110 & 2-Methyl-1-propanol & S, MS & Fusel, bitter & $146.7 \pm 2.3$ & $14.8 \pm 8.0$ & n.d & $2.0 \pm 1.0$ \\
\hline 7 & 8.107 & 1125 & 1121 & Isoamyl acetate & MS & Banana, glue-like & $7.2 \pm 1.4$ & $2.0 \pm 1.9$ & n.d & n.d \\
\hline 8 & 8.715 & 1159 & 1153 & 1-Butanol ${ }^{\mathrm{m}}$ & S, MS & Fruity, medicine & $2.4 \pm 0.2$ & n.d & n.d & n.d \\
\hline 9 & 12.101 & 1220 & 1210 & 3-Methyl-1-butanol & S, MS & Fusel, bitter, harsh & $1317.3 \pm 1.7$ & $170.5 \pm 0.9$ & n.d & $20.4 \pm 3.3$ \\
\hline 10 & 13.491 & 1239 & 1229 & Ethyl hexanoate & S, MS & Apple, fruity, anise & $4.4 \pm 0.6$ & $1.6 \pm 4.3$ & n.d & n.d \\
\hline 12 & 14.772 & & 1220 & 3-Ethyl-3-methylheptane & MS & & n.d & n.d & n.d & $0.4 \pm 3.6$ \\
\hline 13 & 17.024 & & 1260 & Ethyl ethoxyacetate $^{\mathrm{m}}$ & MS & & $3.6 \pm 7.1$ & n.d & n.d & n.d \\
\hline 14 & 19.806 & & 1304 & Ethyl 3-ethoxypropanoate & MS & & n.d & $0.7 \pm 2.4$ & n.d & n.d \\
\hline 15 & 20.230 & 1348 & 1345 & Ethyl lactate & S, MS & Fruity & $546.1 \pm 0.8$ & $73.4 \pm 2.7$ & n.d & $11.8 \pm 6.1$ \\
\hline 16 & 21.005 & 1351 & 1352 & 1-Hexanol & S, MS & Green, grass & $43.8 \pm 1.5$ & $4.9 \pm 1.2$ & n.d & n.d \\
\hline 17 & 22.875 & 1367 & 1364 & (Z)-3-hexen-1-ol ${ }^{\mathrm{m}}$ & MS & Grass, cypress, piney & $5.7 \pm 3.5$ & n.d & n.d & n.d \\
\hline 18 & 24.189 & 1387 & 1384 & Ethyl-2-hydroxy butyrate & MS & Caramel & $2.0 \pm 8.7$ & n.d & n.d & n.d \\
\hline 19 & 25.235 & & 1389 & Ethyl 2-hydroxy acetate & MS & & $7.2 \pm 1.6$ & $0.6 \pm 1.7$ & n.d & n.d \\
\hline 20 & 25.630 & 1383 & 1394 & $\mathrm{EHMB}^{\mathrm{g}, \mathrm{m}}$ & MS & Ripened pineapple, fruity & $14.8 \pm 3.7$ & n.d & n.d & n.d \\
\hline 21 & 26.477 & 1423 & 1428 & $c^{2}$-linalooloxide ${ }^{\mathrm{m}}$ & MS & Sweet, floral, creamy & $4.7 \pm 4.6$ & n.d & n.d & n.d \\
\hline 22 & 27.500 & 1452 & 1447 & Acetic acid & S, MS & Vinegar, pungent & $8.1 \pm 1.9$ & $3.7 \pm 1.7$ & n.d & $0.9 \pm 1.1$ \\
\hline 23 & 28.114 & & 1454 & Isobutyl lactate ${ }^{\mathrm{m}}$ & MS & & $5.1 \pm 1.0$ & n.d & n.d & n.d \\
\hline 24 & 28.431 & 1476 & 1468 & 2-Furfural & S, MS & Sweet, wood & $77.7 \pm 2.1$ & $7.1 \pm 4.1$ & n.d & n.d \\
\hline 26 & 31.089 & 1490 & 1482 & 2-Acetylfuran & MS & Balsamic, caramel, sweet & $7.9 \pm 0.7$ & n.d & n.d & n.d \\
\hline 27 & 31.841 & 1495 & 1493 & Benzaldehyde & S, MS & Almond, burnt sugar & $58.5 \pm 4.0$ & $8.5 \pm 2.2$ & $13.2 \pm 4.0$ & n.d \\
\hline 28 & 33.799 & 1556 & 1523 & 2,3-Butanediol & S, MS & Fruity, onion & $7.7 \pm 5.1$ & $1.5 \pm 3.9$ & n.d & n.d \\
\hline 29 & 34.031 & 1551 & 1542 & Ethyl dl-2-hydroxyhexanoate & MS & Green, fruity, fresh & $16.9 \pm 5.5$ & $1.7 \pm 4.7$ & n.d & n.d \\
\hline 30 & 35.296 & 1550 & 1563 & 1-Octanol & S, MS & Pleasant, sweet & n.d & $1.6 \pm 3.3$ & n.d & $1.0 \pm 6.1$ \\
\hline 31 & 35.698 & 1558 & 1568 & Isoamyl lactate & MS & Creamy, nutty & $12.2 \pm 6.2$ & $2.0 \pm 1.3$ & n.d & n.d \\
\hline 32 & 35.841 & 1572 & 1574 & 5-Methyl-2-furfural & S, MS & Caramel, burnt sugar & $16.6 \pm 5.8$ & $1.1 \pm 1.4$ & n.d & n.d \\
\hline 33 & 38.613 & & 1595 & Ethyllevulate & MS & Sweet, fruity, cherry, & $89.9 \pm 4.4$ & $5.9 \pm 2.6$ & n.d & $0.3 \pm 3.3$ \\
\hline 34 & 39.424 & 1596 & 1608 & Ethyl 2-furoate & MS & Woody, oily & $10.5 \pm 3.2$ & $1.0 \pm 2.8$ & n.d & n.d \\
\hline 35 & 39.725 & 1605 & 1614 & Butyric acid ${ }^{\mathrm{m}}$ & S, MS & Cheese, rancid, sweat & $7.7 \pm 1.7$ & n.d & n.d & n.d \\
\hline 36 & 40.218 & 1635 & 1623 & Phenylacetaldehyde & S, MS & Honey, floral, sweet & n.d & n.d & $1.0 \pm 7.8$ & n.d \\
\hline 37 & 40.532 & 1645 & 1629 & Acetophenone & S, MS & Jasmine, almond & n.d & n.d & $2.5 \pm 1.9$ & n.d \\
\hline 38 & 41.097 & & 1639 & Diethyl methyl succinate ${ }^{\mathrm{m}}$ & S, MS & Fruity, wine-like & $18.7 \pm 4.8$ & n.d & n.d & n.d \\
\hline 39 & 41.741 & 1648 & 1650 & Ethyl benzoate & S, MS & Camomile, celery & $10.6 \pm 5.0$ & $1.7 \pm 3.1$ & n.d & n.d \\
\hline 40 & 43.827 & 1684 & 1686 & Diethyl succinate & MS & Wine, fruity, watermelon & $1349.4 \pm 5.3$ & $213.9 \pm 4.2$ & $6.0 \pm 3.7$ & $12.7 \pm 1.0$ \\
\hline 41 & 44.291 & & 1694 & 3-Ethyl benzaldehyde & $\mathrm{S}, \mathrm{MS}$ & Almond, sweet & n.d & n.d & $1.0 \pm 0.7$ & n.d \\
\hline 42 & 44.307 & & 1695 & 4-Ethyl benzaldehyde & MS & & n.d & $4.6 \pm 2.7$ & n.d & n.d \\
\hline 43 & 45.582 & 1665 & 1719 & $\gamma$-Ethoxybutyrolactone $\mathrm{e}^{\mathrm{m}}$ & MS & & $6.9 \pm 2.9$ & n.d & n.d & n.d \\
\hline 44 & 48.347 & 1789 & 1772 & Ethyl propyl succinate ${ }^{\mathrm{m}}$ & MS & & $4.9 \pm 6.3$ & n.d & n.d & n.d \\
\hline 45 & 49.942 & 1782 & 1801 & Ethyl phenylacetate & MS & Sweet, flowery, rose & $7.7 \pm 5.0$ & $1.0 \pm 0.9$ & n.d & n.d \\
\hline 46 & 50.093 & & 1804 & Diethyl glutarate & MS & & $10.5 \pm 5.1$ & $1.2 \pm 7.8$ & n.d & n.d \\
\hline 47 & 50.393 & & 1810 & 1-(2-Butoxyethoxy)-ethanol ${ }^{\mathrm{m}}$ & MS & & $7.7 \pm 3.5$ & n.d & n.d & n.d \\
\hline 48 & 52.188 & & 1845 & $m$-Ethylacetophenone & MS & Strong, acacia & n.d & $7.7 \pm 4.1$ & n.d & n.d \\
\hline 49 & 53.986 & 1867 & 1879 & Hexanoic acid & S, MS & Cheese, fatty & $16.1 \pm 1.8$ & $0.04 \pm 3.5$ & n.d & n.d \\
\hline 50 & 54.291 & & 1885 & $p$-Ethylacetophenone & MS & Hawthorn, fresh, floral & n.d & $13.5 \pm 4.7$ & n.d & n.d \\
\hline 51 & 54.489 & & 1888 & DEHMS $^{\mathrm{h}, \mathrm{m}}$ & MS & & $46.8 \pm 1.1$ & n.d & n.d & n.d \\
\hline 52 & 55.584 & 1881 & 1909 & Benzyl alcohol & S, MS & Floral, raspberry & $36.8 \pm 3.8$ & $2.0 \pm 1.9$ & n.d & n.d \\
\hline 53 & 57.794 & 1931 & 1951 & 2-Phenylethanol & S, MS & Roses, honey, pollen & $1116.5 \pm 9.9$ & $114.2 \pm 2.3$ & $2.5 \pm 1.7$ & $12.2 \pm 4.7$ \\
\hline 54 & 59.784 & 1977 & 1987 & (E)-whiskey lactone & MS & Coconut, flowery & $41.0 \pm 5.8$ & $5.2 \pm 3.3$ & n.d & n.d \\
\hline 55 & 62.102 & 1944 & 2028 & 2,5-Furandicarboxaldehyde ${ }^{\mathrm{m}}$ & S, MS & & $31.5 \pm 7.8$ & n.d & n.d & n.d \\
\hline
\end{tabular}


Table 3 (Continued)

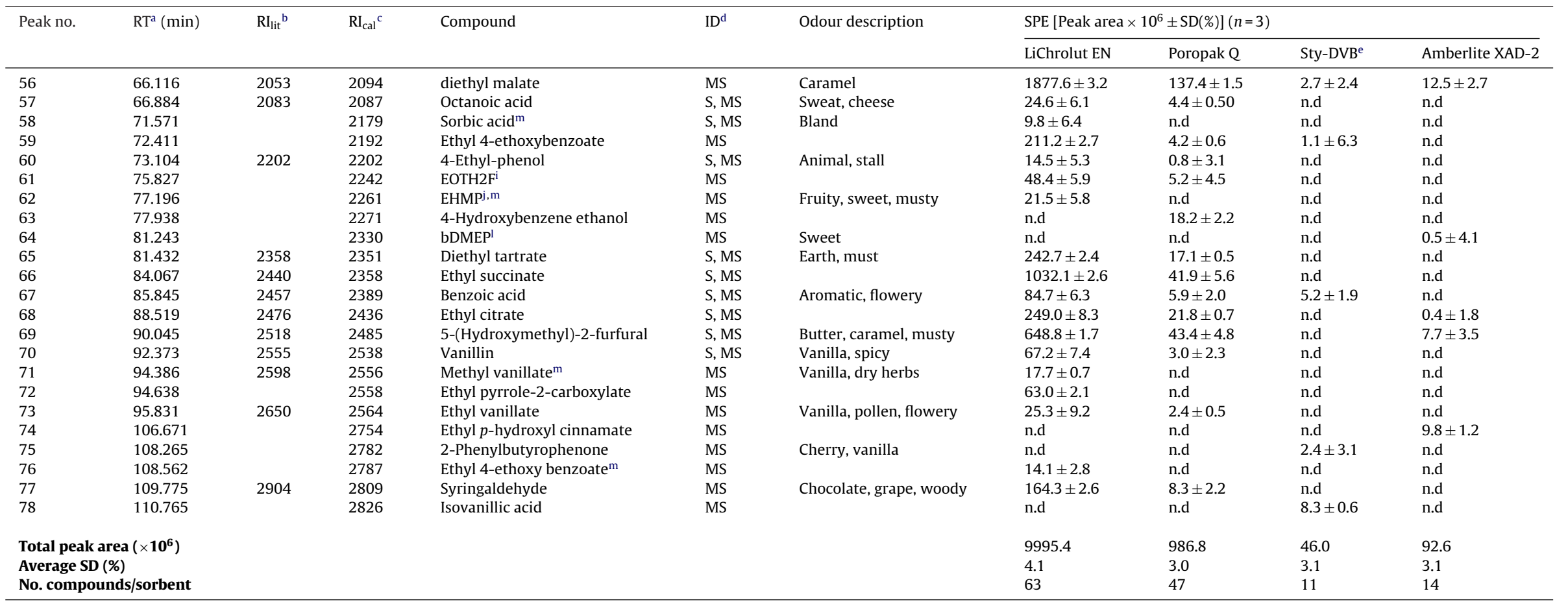

a Retention time (min).

b Retention indices reported in the literature for BP-20 capillary column or equivalents [51-61].

${ }^{c}$ Retention indices calculated from $\mathrm{C}_{8}$ to $\mathrm{C}_{20} n$-linear alkanes with BP-20 capillary column.

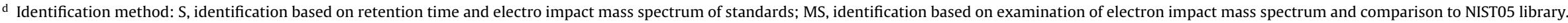

e Styrene-DVB.

${ }^{\mathrm{f}}$ Not detected.

g Ethyl 2-hydroxy-3-methyl-butyrate.

h Diethyl 2-hydroxy-3-methylsuccinate.

${ }^{i}$ Ethyl 5-oxotetrahydro-2-furancarboxylate.

${ }^{\mathrm{j}}$ Ethyl 2-hydroxy-4-methyl-pentanoate.

1 2,5-Bis(1,1-dimethylethyl)-phenol.

m VOCs and SVOCs extracted only by LiChrolut EN sorbent. 
Table 4

Volatile and semi-volatile composition of Bual Madeira wine obtained by MEPS/GC-qMS methodology, the corresponding retention times, retention indices, and odour description.

\begin{tabular}{|c|c|c|c|c|c|c|c|c|c|c|c|}
\hline \multirow[t]{2}{*}{ Peak no. } & \multirow[t]{2}{*}{$\mathrm{RT}^{\mathrm{a}}(\min )$} & \multirow[t]{2}{*}{$\mathrm{RI}_{\mathrm{lit}}^{\mathrm{b}}$} & \multirow[t]{2}{*}{$\mathrm{RI}_{\mathrm{cal}} \mathrm{C}^{\mathrm{c}}$} & \multirow[t]{2}{*}{ Compound } & \multirow[t]{2}{*}{$\mathrm{ID}^{\mathrm{d}}$} & \multirow[t]{2}{*}{ Odour description } & \multicolumn{5}{|c|}{ MEPS $\left[\right.$ Peak area $\left.\times 10^{6} \pm \operatorname{SD}(\%)\right](n=3)$} \\
\hline & & & & & & & $\mathrm{C}_{18}$ & $\mathrm{C}_{8}$ & $\mathrm{C}_{2}$ & SIL & M1 \\
\hline 1 & 5.337 & $1099^{g}$ & 1118 & 2-Methyl-1-propanol & S, MS & Fusel, bitter & $1.3 \pm 4.0$ & $1.3 \pm 1.1$ & n.d $\mathrm{d}^{\mathrm{e}}$ & n.d & $1.0 \pm 6.8$ \\
\hline 2 & 8.696 & $1220^{g}$ & 1202 & 3-Methyl-1-butanol & S, MS & Fusel, bitter, harsh & $27.3 \pm 2.2$ & $26.2 \pm 0.06$ & $1.8 \pm 1.0$ & $0.9 \pm 4.3$ & $19.2 \pm 1.9$ \\
\hline 3 & 13.674 & 1348 & 1340 & Ethyl lactate & S, MS & Fruity & $6.2 \pm 2.7$ & $9.0 \pm 0.6$ & $1.8 \pm 0.6$ & $1.4 \pm 4.1$ & $6.5 \pm 2.2$ \\
\hline 4 & 14.079 & 1351 & 1336 & 1-Hexanol & S, MS & Green, grass & $1.1 \pm 2.6$ & $0.7 \pm 9.5$ & n.d & n.d & $0.6 \pm 0.8$ \\
\hline 5 & 18.165 & $1452^{\mathrm{g}}$ & 1469 & Acetic acid & S, MS & Vinegar, pungent & $0.8 \pm 3.7$ & $0.3 \pm 2.2$ & n.d & n.d & $0.2 \pm 1.3$ \\
\hline 6 & 24.503 & & 1579 & Ethyllevulate & MS & Sweet, fruity, cherry & $0.3 \pm 5.8$ & $0.1 \pm 2.7$ & n.d & n.d & $0.2 \pm 0.4$ \\
\hline 7 & 27.219 & & 1621 & Diethyl succinate & S, MS & Fruity, wine-like & $25.4 \pm 1.8$ & $25.5 \pm 0.8$ & $1.1 \pm 0.3$ & $0.1 \pm 1.6$ & $20.0 \pm 1.1$ \\
\hline 8 & 35.698 & 1931 & 1953 & 2-Phenylethanol & S, MS & Roses, honey, pollen & $12.3 \pm 2.4$ & $11.7 \pm 0.9$ & $0.3 \pm 5.1$ & n.d & $9.1 \pm 0.9$ \\
\hline 9 & 40.343 & $2053^{\mathrm{h}}$ & 2075 & Diethyl malate & S, MS & Caramel & $16.6 \pm 1.6$ & $20.0 \pm 0.9$ & $2.2 \pm 0.4$ & $0.9 \pm 4.7$ & $16.1 \pm 0.9$ \\
\hline 10 & 44.363 & & 2196 & Ethyl 4-ethoxybenzoate & MS & & $1.6 \pm 3.5$ & $0.7 \pm 2.6$ & n.d & n.d & $0.7 \pm 4.5$ \\
\hline 11 & 46.288 & & 2233 & $\mathrm{HMBL}^{\mathrm{j}}$ & S, MS & & $0.50 \pm 0.7$ & $0.6 \pm 9.3$ & n.d & n.d & $0.5 \pm 6.9$ \\
\hline 12 & 48.972 & & 2330 & bDMEP $P^{1}$ & MS & Sweet & $0.2 \pm 1.2$ & n.d & n.d & $0.6 \pm 0.1$ & $0.1 \pm 0.4$ \\
\hline 13 & 50.943 & 2440 & 2388 & Ethyl succinate & S, MS & & $0.8 \pm 4.1$ & $0.6 \pm 1.1$ & n.d & n.d & n.d \\
\hline 14 & 53.404 & $2476^{\mathrm{h}}$ & 2438 & Ethyl citrate & S, MS & & $3.9 \pm 1.8$ & $4.0 \pm 0.2$ & $0.2 \pm 8.7$ & $0.4 \pm 1.9$ & $3.2 \pm 0.6$ \\
\hline 15 & 54.293 & $2518^{\mathrm{h}}$ & 2489 & 5-(Hydrxoymethyl)-2-furfural & S, MS & Butter, caramel, musty & $3.3 \pm 3.0$ & $5.9 \pm 1.6$ & $1.8 \pm 2.1$ & $7.1 \pm 0.9$ & $4.6 \pm 0.1$ \\
\hline \multirow{2}{*}{\multicolumn{2}{|c|}{$\begin{array}{l}\text { Total peak area }\left(\times 10^{6}\right) \\
\text { Average SD }(\%)\end{array}$}} & & & & & & 101.7 & 106.7 & 9.8 & 11.3 & 81.9 \\
\hline & & & & & & & 2.8 & 2.4 & 2.6 & 2.5 & 2.1 \\
\hline \multicolumn{2}{|c|}{ No. compounds/sorbent } & & & & & & 15 & 14 & 8 & 7 & 14 \\
\hline
\end{tabular}

a Retention time ( $\mathrm{min}$ )

b Retention indices reported in the literature for BP-20 capillary column or equivalents [51-61]

c Retention indices calculated from $C_{8}$ to $C_{20} n$-linear alkanes.

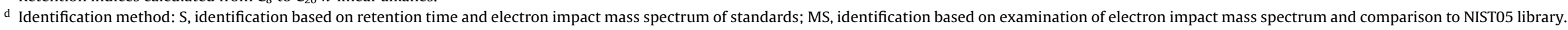

e Not detected.

g Reference [53].

${ }^{\text {h }}$ Reference [60,61].

j 5-Hydroxymethyl $\gamma$-butyrolactone.

1 2,5-Bis(1,1-dimethyl)-phenol. 
Table 5

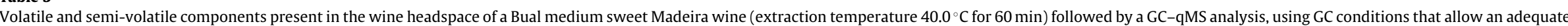
chromatographic resolution, the corresponding retention times, retention indices, and odour description.

\begin{tabular}{|c|c|c|c|c|c|c|c|c|c|c|c|c|}
\hline \multirow[t]{2}{*}{ Peak no. } & \multirow[t]{2}{*}{$\mathrm{RT}^{\mathrm{a}}(\min )$} & \multirow[t]{2}{*}{$\mathrm{RI}_{\mathrm{lit}}^{\mathrm{b}}$} & \multirow[t]{2}{*}{$\mathrm{RI}_{\mathrm{cal}}^{\mathrm{c}}$} & \multirow[t]{2}{*}{ Compound } & \multirow[t]{2}{*}{$\mathrm{ID}^{\mathrm{d}}$} & \multirow[t]{2}{*}{ Odour description } & \multicolumn{6}{|c|}{ SPME $\left(\right.$ Peak area $\left.\times 10^{6} \pm \operatorname{RSD}(\%)\right)(n=3)$} \\
\hline & & & & & & & DVB/CAR/PDMS & CAR/PDMS & PDMS/DVB & PDMS & PA & PEG \\
\hline 1 & 7.365 & 1099 & 1110 & 2-Methyl-1-propanol & S, MS & Fusel, bitter, & $34.0 \pm 1.1$ & $13.7 \pm 1.0$ & $13.7 \pm 1.5$ & n.d & $24.8 \pm 1.3$ & $22.4 \pm 3.2$ \\
\hline 2 & 9.735 & 1201 & 1126 & Limonene & S, MS & Citrus-like, fresh & $37.3 \pm 2.1$ & $12.6 \pm 3.5$ & $10.8 \pm 3.4$ & $7.0 \pm 4.8$ & $1.8 \pm 2.5$ & $0.6 \pm 0.86$ \\
\hline 3 & 10.637 & & 1145 & (E)-6-dodecene & MS & & $2.0 \pm 0.4$ & n.d $\mathrm{d}^{\mathrm{e}}$ & n.d & n.d & n.d & n.d \\
\hline 4 & 11.853 & & 1168 & 1-Dodecene & MS & & n.d & n.d & n.d & $10.7 \pm 0.8$ & n.d & n.d \\
\hline 5 & 12.644 & 1220 & 1210 & 3-Methyl-1-butanol & S, MS & Fusel, bitter, harsh & $354.3 \pm 0.6$ & $420.6 \pm 0.7$ & $236.8 \pm 0.9$ & $195.2 \pm 1.3$ & $242 . \pm 0.84$ & $294.4 \pm 1.6$ \\
\hline 6 & 12.942 & 1239 & 1229 & Ethyl hexanoate & S, MS & Apple, fruity, anise & n.d & $15.3 \pm 4.3$ & n.d & n.d & n.d & n.d \\
\hline 7 & 13.347 & & 1232 & (E)-3-dodecene & MS & & $2.1 \pm 0.3$ & n.d & n.d & n.d & n.d & n.d \\
\hline 8 & 14.007 & & 1240 & (Z)-2-dodecene & MS & & $5.1 \pm 0.4$ & n.d & $2.6 \pm 0.4$ & $3.1 \pm 0.9$ & n.d & n.d \\
\hline 9 & 14.501 & 1263 & 1245 & $p$-Cymene & MS & Spicy, herbaceous, citrus-like & n.d & $7.7 \pm 0.73$ & n.d & n.d & n.d & n.d \\
\hline 10 & 19.567 & & 1304 & Ethyl 3-ethoxypropanoate & MS & & $3.0 \pm 6.7$ & $3.3 \pm 1.5$ & $1.7 \pm 1.7$ & n.d & n.d & n.d \\
\hline 11 & 20.368 & 1348 & 1345 & Ethyl lactate & MS & Fruity & $53.9 \pm 3.7$ & $58.1 \pm 1.0$ & $49.8 \pm 1.7$ & $53.9 \pm 4.2$ & $44.7 \pm 0.8$ & $72.0 \pm 0.8$ \\
\hline 12 & 21.061 & 1351 & 1352 & 1-Hexanol & S, MS & Green, grass & $15.8 \pm 1.4$ & $39.9 \pm 1.5$ & $8.0 \pm 7.4$ & $8.7 \pm 1.3$ & $8.1 \pm 0.6$ & $7.7 \pm 1.0$ \\
\hline 13 & 22.908 & 1367 & 1364 & (Z)-3-Hexen-1-ol & S, MS & Grass, cypress, piney & n.d & $1.4 \pm 1.02$ & n.d & n.d & n.d & n.d \\
\hline 14 & 23.002 & 1385 & 1373 & Nonanal & MS & Citrus, fatty & $3.4 \pm 1.42$ & n.d & $2.0 \pm 2.71$ & n.d & n.d & n.d \\
\hline 15 & 23.471 & 1400 & 1379 & Tetradecane & MS & & n.d & n.d & n.d & $5.5 \pm 4.3$ & n.d & n.d \\
\hline 16 & 25.634 & 1383 & 1395 & EHMB $^{\mathrm{f}}$ & MS & Ripened pineapple, fruity & $1.9 \pm 2.38$ & $1.4 \pm 1.00$ & n.d & n.d & n.d & n.d \\
\hline 17 & 26.111 & 1436 & 1402 & Ethyl octanoate & S, MS & Fruity, fat & $5.5 \pm 3.8$ & n.d & $4.6 \pm 4.9$ & $7.4 \pm 1.4$ & $1.0 \pm 2.4$ & n.d \\
\hline 18 & 26.683 & & 1412 & 1-Tetradecene & MS & & $5.3 \pm 0.6$ & n.d & $8.6 \pm 2.51$ & $11.7 \pm 6.0$ & n.d & n.d \\
\hline 20 & 27.729 & 1452 & 1447 & Acetic acid & S, MS & Vinegar, pungent & $5.6 \pm 1.4$ & $3.6 \pm 1.1$ & $5.7 \pm 2.8$ & $15.9 \pm 5.0$ & $11.8 \pm 1.5$ & $35.8 \pm 0.6$ \\
\hline 21 & 28.468 & 1476 & 1468 & 2-Furfural & S, MS & Sweet, wood & $62.2 \pm 0.4$ & $100.7 \pm 0.7$ & $6.7 \pm 3.3$ & $12.7 \pm 6.8$ & $6.1 \pm 0.85$ & $7.8 \pm 1.4$ \\
\hline 22 & 30.399 & 1484 & 1471 & Decanal & MS & Tallow, soap, orange & n.d & n.d & $10.5 \pm 3.9$ & $16.6 \pm 3.2$ & n.d & n.d \\
\hline 23 & 30.504 & & 1473 & Isooctanol & MS & & $9.1 \pm 0.1$ & $2.8 \pm 3.4$ & n.d & n.d & $7.2 \pm 0.4$ & $1.1 \pm 0.4$ \\
\hline 24 & 31.096 & 1490 & 1482 & 2-Acetylfuran & MS & Balsamic, caramel & n.d & $2.7 \pm 0.4$ & n.d & n.d & n.d & n.d \\
\hline 25 & 31.243 & & 1484 & Ethyl sorbate & MS & Fruity, sweet, pineapple & $3.3 \pm 0.14$ & n.d & $2.2 \pm 3.3$ & n.d & n.d & n.d \\
\hline 26 & 31.828 & 1495 & 1493 & Benzaldehyde & S, MS & Almond, burnt sugar & $91.1 \pm 0.4$ & $86.7 \pm 1.1$ & $9.1 \pm 0.2$ & $8.7 \pm 2.2$ & $6.6 \pm 0.2$ & $5.6 \pm 4.2$ \\
\hline 27 & 33.952 & & 1526 & 2,6-Dimethyl-4-heptanol & MS & & $3.7 \pm 0.1$ & $2.2 \pm 0.6$ & $3.7 \pm 1.3$ & n.d & n.d & n.d \\
\hline 28 & 34.047 & 1556 & 1523 & 2,3-Butanediol & MS & Fruity, onion & n.d & n.d & n.d & n.d & n.d & $4.8 \pm 0.9$ \\
\hline 29 & 35.838 & 1572 & 1574 & 5-Methyl-2-furfural & MS & Caramel, burnt sugar & $13.9 \pm 0.7$ & $7.5 \pm 0.2$ & n.d & n.d & n.d & n.d \\
\hline 30 & 38.568 & & 1595 & Ethyllevulate & MS & Sweet, fruity, cherry & $3.2 \pm 0.6$ & $3.7 \pm 5.3$ & $3.5 \pm 0.6$ & $5.3 \pm 1.2$ & $2.3 \pm 2.9$ & $2.0 \pm 0.8$ \\
\hline 31 & 38.741 & & 1596 & $\gamma$-Butyrolactone & MS & Cheese, burnt sugar & n.d & n.d & n.d & n.d & $1.0 \pm 0.8$ & n.d \\
\hline 32 & 38.983 & & 1600 & Carbitol & MS & & n.d & n.d & n.d & n.d & $3.6 \pm 0.6$ & $2.7 \pm 3.3$ \\
\hline 33 & 39.346 & 1596 & 1608 & Ethyl 2-furoate & MS & Woody, oily & $9.0 \pm 0.2$ & $6.4 \pm 4.4$ & $3.2 \pm 0.9$ & $4.2 \pm 1.5$ & $1.6 \pm 2.4$ & $1.4 \pm 2.5$ \\
\hline 34 & 41.003 & & 1639 & Diethyl methylsuccinate & S, MS & Fruity, wine-like & $3.0 \pm 0.4$ & n.d & $3.0 \pm 1.4$ & n.d & $0.7 \pm 0.7$ & n.d \\
\hline 35 & 41.614 & 1648 & 1650 & Ethyl benzoate & S, MS & Camomile, celery & $21.1 \pm 0.5$ & $6.4 \pm 2.4$ & $12.4 \pm 3.9$ & n.d & $4.5 \pm 1.0$ & $2.5 \pm 1.6$ \\
\hline 36 & 43.425 & 1684 & 1686 & diethyl succinate & S, MS & Wine, fruity, watermelon & $203.1 \pm 0.1$ & $124.3 \pm 0.5$ & $266.3 \pm 1.3$ & $129.8 \pm 2.4$ & $120 \pm 2.4$ & $78.6 \pm 0.8$ \\
\hline 37 & 49.828 & 1782 & 1801 & Ethyl phenylacetate & MS & Sweet, flowery, rose & $10.6 \pm 3.1$ & $1.7 \pm 0.7$ & $10.7 \pm 6.3$ & $9.3 \pm 0.3$ & $2.8 \pm 1.5$ & $1.4 \pm 0.4$ \\
\hline 38 & 50.894 & & 1820 & 3,4-Dimethyl-benzaldehyde & MS & & $8.8 \pm 0.9$ & $1.2 \pm 0.5$ & $9.9 \pm 0.9$ & n.d & $2.3 \pm 1.0$ & n.d \\
\hline 39 & 51.724 & & 1836 & 2-Phenylethyl acetate & MS & roses & n.d & n.d & n.d & n.d & $0.9 \pm 0.75$ & n.d \\
\hline 40 & 54.456 & & 1888 & DEHMS ${ }^{g}$ & MS & & n.d & n.d & $7.5 \pm 3.23$ & $21.8 \pm 2.75$ & n.d & n.d \\
\hline 41 & 55.646 & 1881 & 1909 & Benzyl alcohol & S, MS & Floral, raspberry & $4.7 \pm 0.2$ & $0.7 \pm 4.4$ & $6.2 \pm 0.7$ & n.d & $1.6 \pm 0.4$ & $1.6 \pm 1.9$ \\
\hline 42 & 55.669 & & 1911 & TMPOPMP & MS & & n.d & n.d & n.d & $18.6 \pm 2.7$ & n.d & n.d \\
\hline 43 & 57.16 & & 1939 & $\alpha$-Ionol & MS & & $14.4 \pm 0.3$ & $0.7 \pm 0.2$ & $12.8 \pm 0.9$ & $19.2 \pm 0.9$ & $6.5 \pm 0.7$ & $4.3 \pm 1.6$ \\
\hline
\end{tabular}




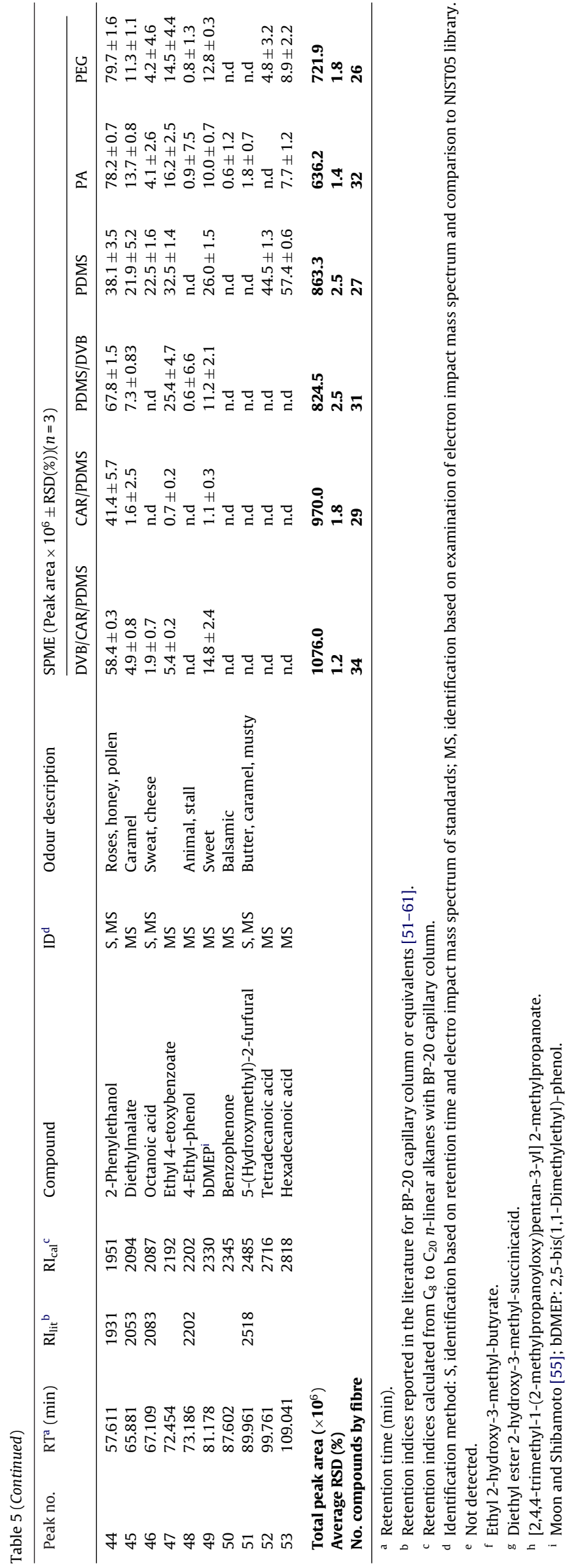

As can be observed, LiChrolut EN was, by far, the sorbent that provided the most globally favourable analytical response, whereas for the other sorbents the sorption capacity was variable ranging from less than $0.5 \%$ (Sty-DVB) to ca. 10\% (Poropak Q). The higher sorption capacity of polymeric LiChrolut EN for Bual wine VOCs and SVOCs, with regards to other sorbents, can be ascribed to specific interactions of this sorbent with relatively polar solutes, in addition to the typical hydrophobic mechanisms. According to Table 3, Poropak Q sorbent present lower potential to extract Bual wine VOCs and SVOCs (47 compounds) than LiChrolut EN, while Amberlite XAD-2 and Sty-DVB sorbent present the lowest extraction efficiency, being able to extract only 14 and 11 compounds, respectively.

Considering the aroma compounds by groups (Fig. 3), the esters and higher alcohols were the predominant volatiles, followed by furan and carbonyl compounds, the two first groups, produced during the alcoholic fermentation, playing an important role on the flavor of wines. C-6 alcohol fraction is easily recognizable by their strong and pungent smell and taste, related to herbaceous notes $[63,64]$. As for the esters, they are regarded as very important in the aroma of young wines due to their fruity flavors [63]. Other minor groups of aroma compounds detected were volatile fatty acids, carbonyl compounds and furan compounds, According to Fig. 3, the nature of the sorbent/coating is an important aspect for the preconcentration of the considered chemical groups since each shows a different extraction profile.

Considering individual compounds, diethyl malate (56; $1877.6 \times 10^{6} \pm 3.2 \%$ ) was the main component found in the $\mathrm{SPE}_{\text {LiChrolut EN }}$ extracts followed by diethyl succinate (40; $\left.1349.4 \times 10^{6} \pm 5.3 \%\right)$, 3-methyl-1-butanol $\left(\mathbf{9} ; 1317.3 \times 10^{6} \pm 1.7 \%\right)$ and 2-phenylethanol (53; $\left.1116.5 \times 10^{6} \pm 9.9 \%\right)$. Moreover 20 VOCs and SVOCs (coded as " $m$ " in Table 3) were extracted only by this sorbent. Poropak $Q$ seems able to extract diethyl succinate $\left(\mathbf{4 0} ; 213.9 \times 10^{6} \pm 4.2 \%\right)$, 4-hydroxybenzene ethanol (63; $\left.18.2 \times 10^{6} \pm 2.2 \%\right), 1$-hexanol $\left(\mathbf{1 6} ; 4.9 \times 10^{6} \pm 1.2 \%\right)$, and ethyl hexanoate $\left(10 ; 1.6 \times 10^{6} \pm 4.3 \%\right)$, better than the other sorbents used. Benzaldehyde (27; $\left.13.2 \times 10^{6} \pm 4.0 \%\right)$, isovanillic acid (78; $\left.8.3 \times 10^{6} \pm 0.6 \%\right)$, diethyl malate $\left(\mathbf{5 6} ; 2.7 \times 10^{6} \pm 2.4 \%\right)$, and benzoic acid $\left(67 ; 5.2 \times 10^{6} \pm 1.9 \%\right)$, had high affinity for Sty-DVB sorbent, while Amberlite XAD-2 exhibit a strong sorption capacity for 3-methyl-1-butanol (9; 20.4 × 106 $\pm 3.3 \%)$, diethyl succinate $\left(\mathbf{4 0} ; 12.7 \times 10^{6} \pm 1.0 \%\right)$, diethyl malate (56; $\left.12.5 \times 10^{6} \pm 2.7\right)$, 2-phenylethanol (53; $\left.12.2 \times 10^{6} \pm 4.7 \%\right)$, and ethyl lactate $\left(15 ; 11.8 \times 10^{6} \pm 6.1 \%\right)$. Ethyl $p$-hydroxycinnamate (74; $\left.9.8 \times 10^{6} \pm 1.2 \%\right)$ was extracted only by using Amberlite XAD-2 as sorbent.

\subsection{MEPS analysis}

Until the present there are no previous studies reporting the use of MEPS to extract VOCs and SVOCs from wine samples. To fulfil this requirement, and although the most common sorbent is $C_{18}$, different types of sorbents, namely $\mathrm{C}_{18}, \mathrm{C}_{8}, \mathrm{C}_{2}$, M1 and SIL (Table 4 and Fig. 4) were evaluated and compared [51-61].

Fig. 4 shows typical chromatograms of VOCs and SVOCs from a Bual wine sample obtained by MEPS using the sorbents under consideration. Table 4 shows the compounds identified in the above five sorbents, along with their retention indices (RI), odour descriptors and peak area. The results shows, that the $C_{18}, C_{8}$ and $M_{1}$ sorbents extracted almost the same VOCs and SVOCs, 15, 14 and 14 , respectively, $C_{8}$ sorbent was able to concentrate larger amount of extracted compounds (Fig. 2). $C_{2}$ and SIL sorbents were found to be the least efficient to extract VOCs and SVOCs from wine samples. Fig. 2 shows the number of VOCs and SVOCs extracted as a function of the sorbent material and also compares the relative average 


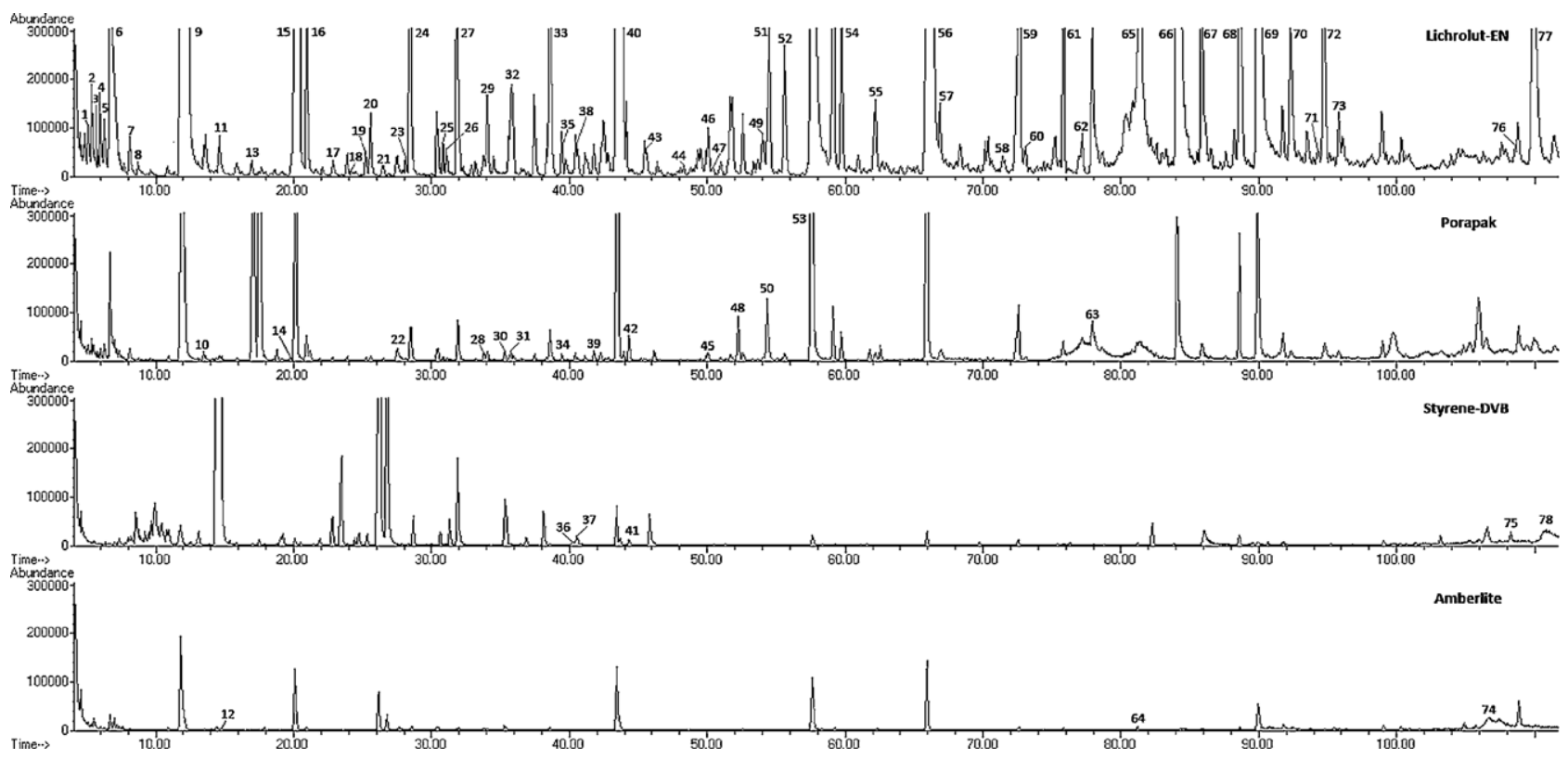

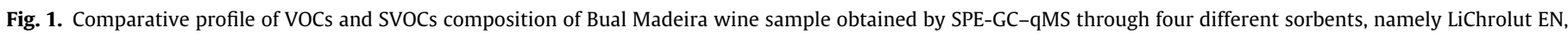
Poropak Q, styrene-DVB and Amberlite XAD-2. Peak assignments and identification appear in Table 3.

percent peak areas obtained for each sorbent, relatively to $C_{8}$ (taken as $100 \%)$.

Considering the identified compounds, the predominant ones isolated through MEPS were 3-methyl-1-butanol (2; $24.2 \pm 3.8 \%$ on average), diethyl succinate $(\mathbf{7} ; 23.2 \pm 5.4 \%)$, diethyl malate (9; $17.9 \pm 6.2 \%), 2$-phenylethanol $(\mathbf{8} ; 10.7 \pm 9.8 \%)$, and ethyl lactate $(\mathbf{3}$; $8.0 \pm 11.2 \%)$, for all sorbents.

As determined by SPE, esters and higher alcohols were the main chemical groups identified by MEPS/GC-qMS. The $\mathrm{C}_{18}$ sorbent revealed higher extraction efficiency to esters and fatty acids, whereas $C_{8}$ is more efficient to extract ethyl esters and furan compounds. For carbonyl compounds and miscellaneous (Mis) SIL present better results (Fig. $3 \mathrm{~b}$ ). Considering the individual VOCs and SVOCs, 5-(hydroxymethyl)-2-furfural and ethyl lactate, were efficiently extracted by SIL, $63 \pm 2.1$ and $12 \pm 4.1 \%$, respectively, of the total fraction extracted by this sorbent. $C_{18}, C_{8}$ and $M 1$ sorbents showed higher potential to extract 3-methyl-1-butanol $\left(27.3 \times 10^{6} \pm 2.2 \% ; \quad 26.2 \times 10^{6} \pm 0.06 \%\right.$, and $19.2 \times 10^{6} \pm 1.9 \%$, respectively, of the total Bual wine VOCs and SVOCs extracted by the corresponding sorbent), diethyl succinate $\left(25.4 \times 10^{6} \pm 1.8 \%\right.$, $25.5 \times 10^{6} \pm 0.8 \%$ and $20.0 \times 10^{6} \pm 1.1 \%$, respectively), diethyl malate $\left(16.6 \times 10^{6} \pm 1.6 \% ; 20.0 \times 10^{6} \pm 0.9 \%\right.$, and $16.1 \times 10^{6} \pm 0.9 \%$, respectively), and 2-phenylethanol $\left(12.3 \times 10^{6} \pm 2.4 \%\right.$; $11.7 \times 10^{6} \pm 0.9 \%$, and $9.1 \times 10^{6} \pm 0.9 \%$, respectively), than $C_{2}$ and SIL materials. $C_{2}$ showed highest efficiency to diethyl malate $\left(2.2 \times 10^{6} \pm 0.4 \%\right)$ and ethyl lactate $\left(1.8 \times 10^{6} \pm 0.6 \%\right)$.

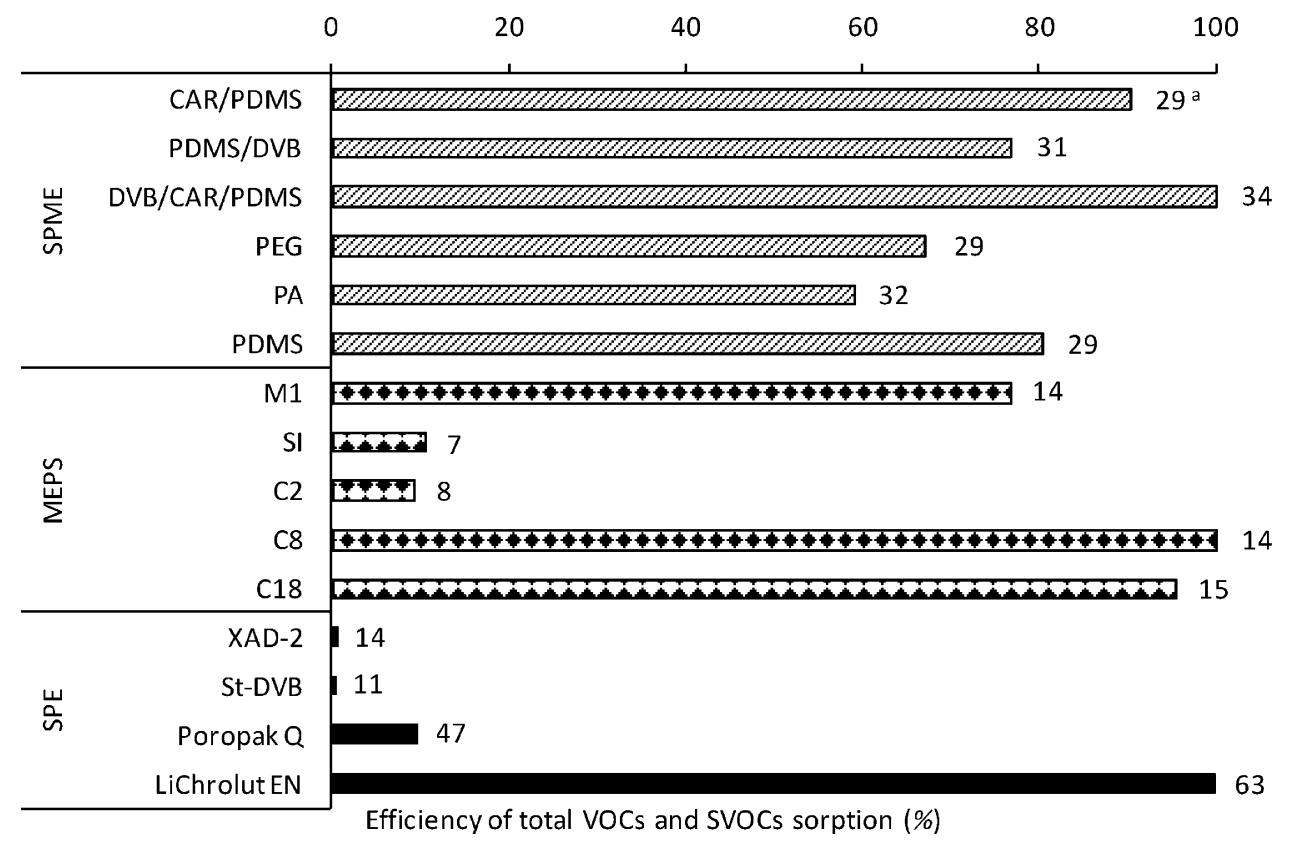

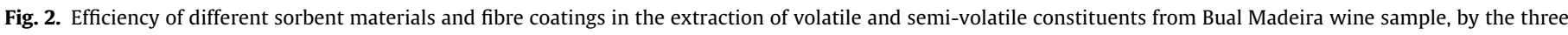

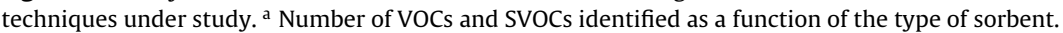



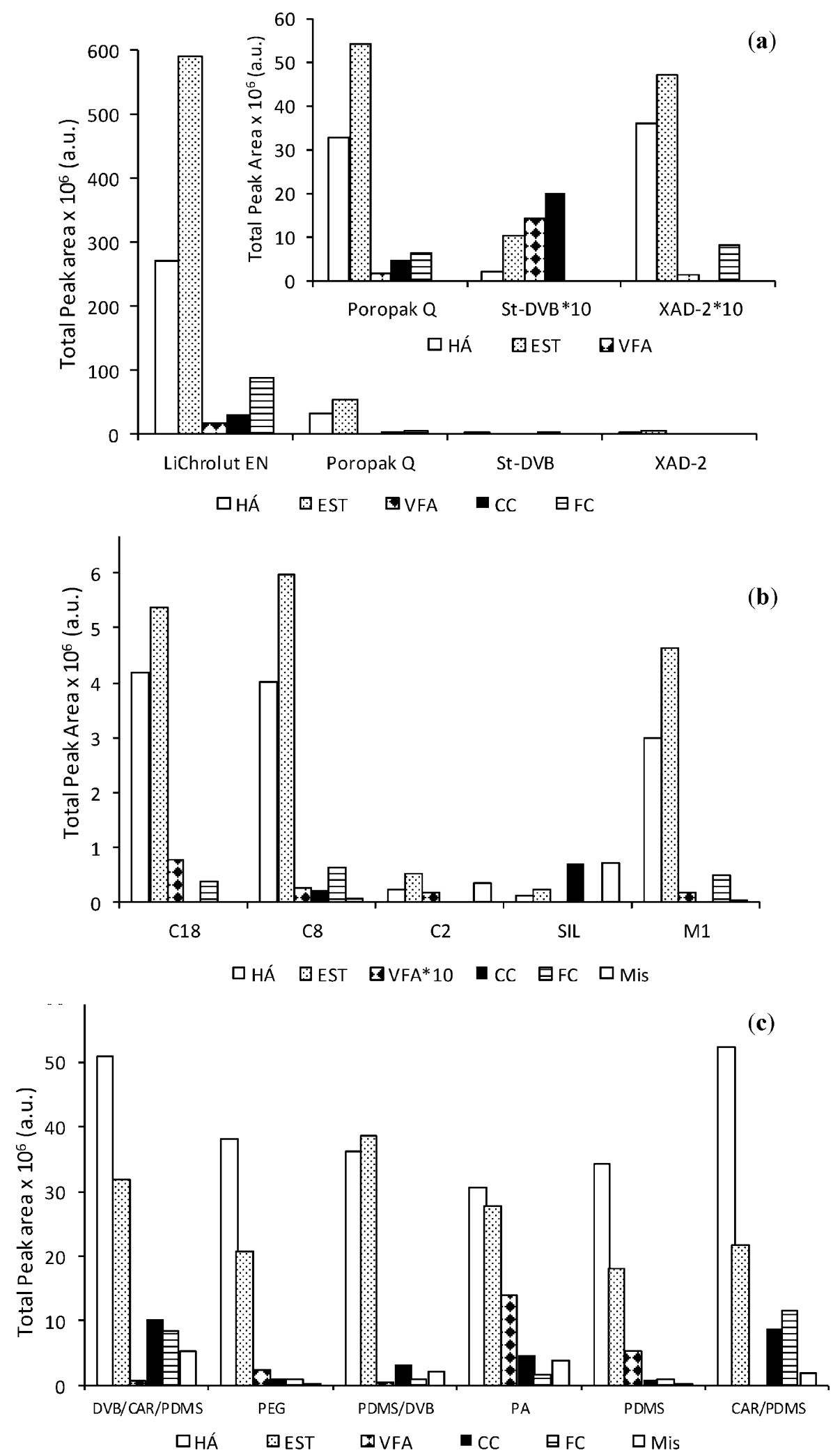

Fig. 3. Comparison of the efficiency of SPE, MEPS and SPME procedures to extract distinct chemical groups found in Bual wine samples.

\subsection{HS-SPME analysis}

The sensitivity of SPME extraction methodology depends greatly on the value of the distribution constant of analytes partitioned between the sample and fibre coating material. To ensure the determination of as many compounds as possible, extraction were performed using six SPME fibres, among the most routinely used for assaying volatiles and semi-volatiles, differing in the polarity and thickness of the stationary phase. They were tested and compared individually in order to find such that the coating having highest affinity towards Bual wine VOCs and SVOCs. The SPME-influencing experimental factors namely extraction time and extraction 


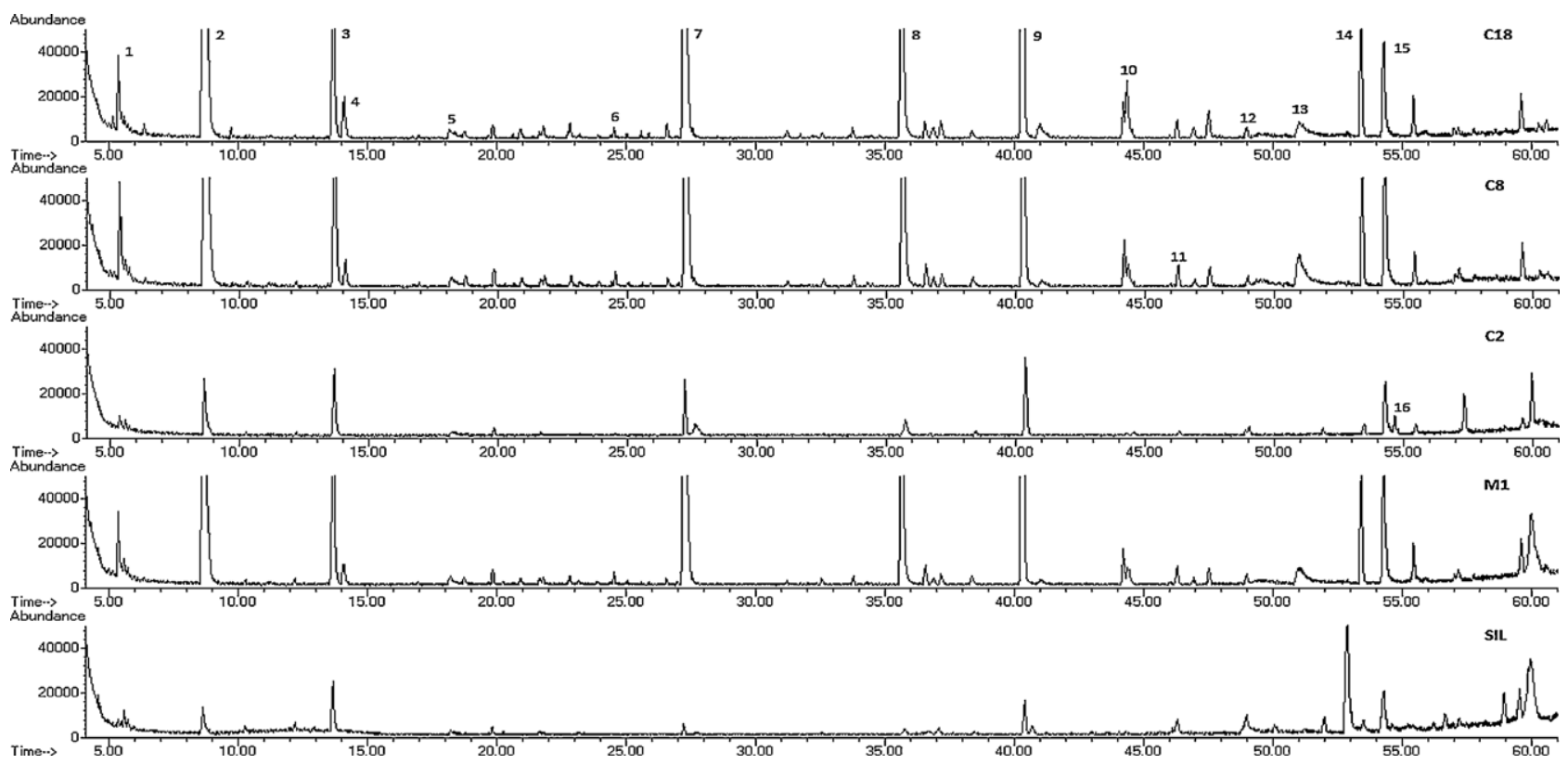

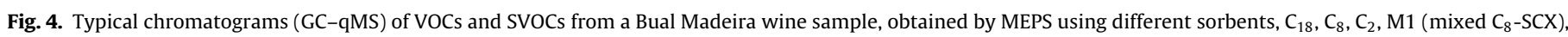
and SIL (silica). Peak assignments and identification appear in Table 4.

temperature, previously evaluated and optimized by Câmara et al. [65], were applied in this work. Wine samples were extracted at a fixed extraction time (60 min) and temperature $\left(40^{\circ} \mathrm{C}\right)$.

The comparison of the SPME fibre performance was performed in terms of total peak areas, number of identifiable compounds in the extract and reproducibility. The chromatographic profiles presented in Fig. 5, obtained for Bual wine using different coatings in same experimental conditions, shows different extraction efficiency of the fibres for the chemical groups identified in wines. The results obtained in rigorously reproduced temperature and exposure time conditions, are reported in Table 5 and Fig. 5 [51-61].

The SPME fibre coated with DVB/CAR/PDMS afforded the highest extraction sensitivity (highest peak areas, highest number of detected compounds and high reproducibility). In contrast and under the same experimental conditions PA fibre present the lowest sorption capacity (Fig. 2; Table 5), although extracts a larger number of VOCs and SVOCs. Fig. 2 also shows the number of VOCs and SVOCs extracted as a function of the type of fibre, and the relative sorption efficiency of the different SPME coatings, taking as $100 \%$ the maximum analytical signal obtained from DVB/CAR/PDMS extract. This procedure allowed the identification of 56 VOCs and SVOCs: 34 with DVB/CAR/PDMS coating, 32 with PA, and 31 with PDMS/DVB. CAR/PMDS, PDMS and PEG extracts a fewer number of volatiles and semi-volatiles, 29, 27 and 26 compounds, respectively (Fig. 2). However the total peak area obtained using CAR/PDMS is significantly higher in comparison with those

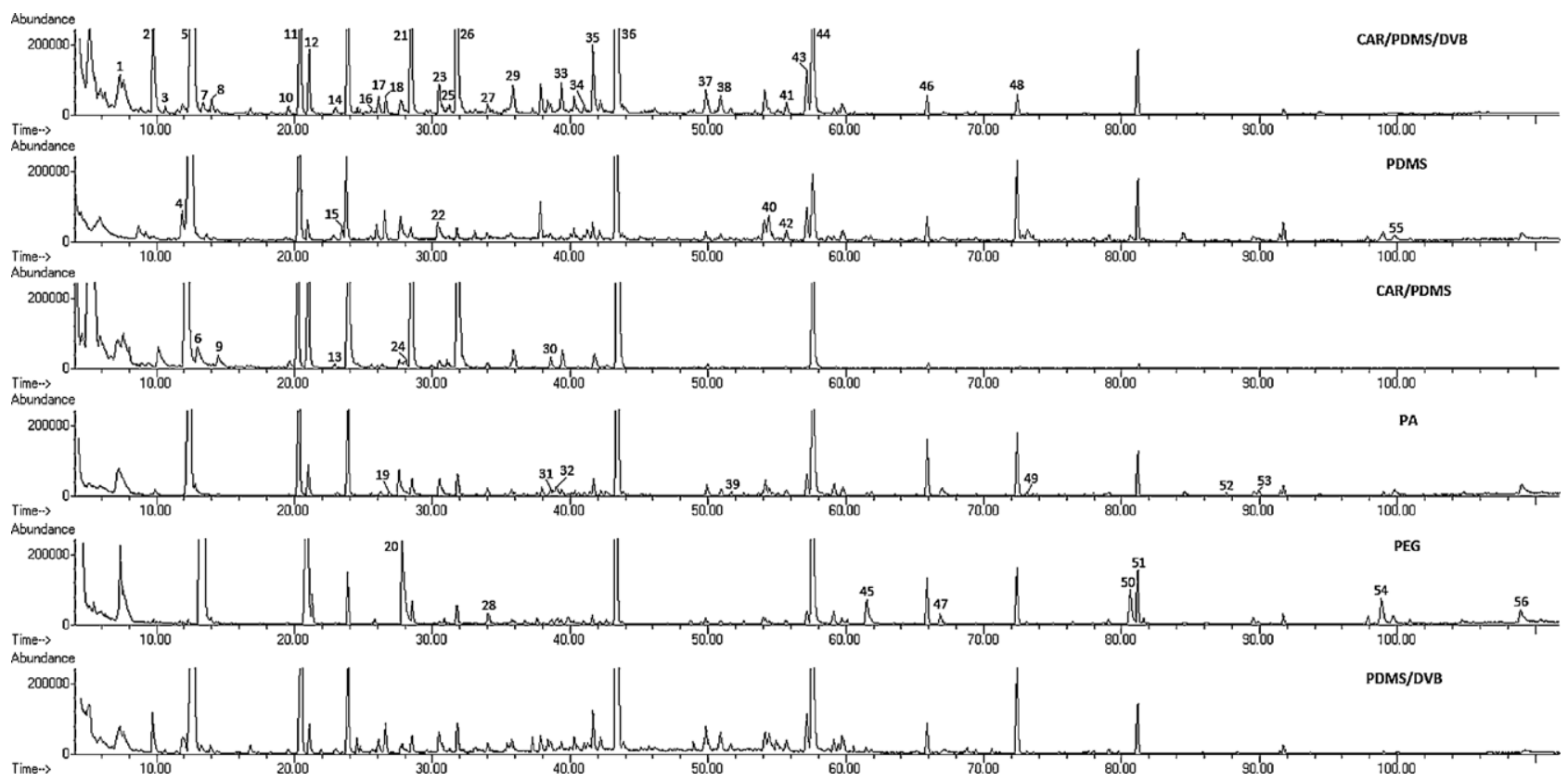

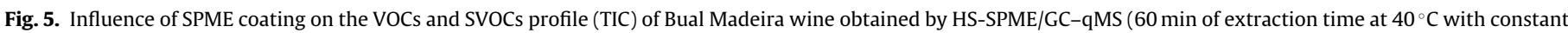
magnetic stirring $(800 \mathrm{rpm})$. Peak assignments and identification appear in Table 5. 

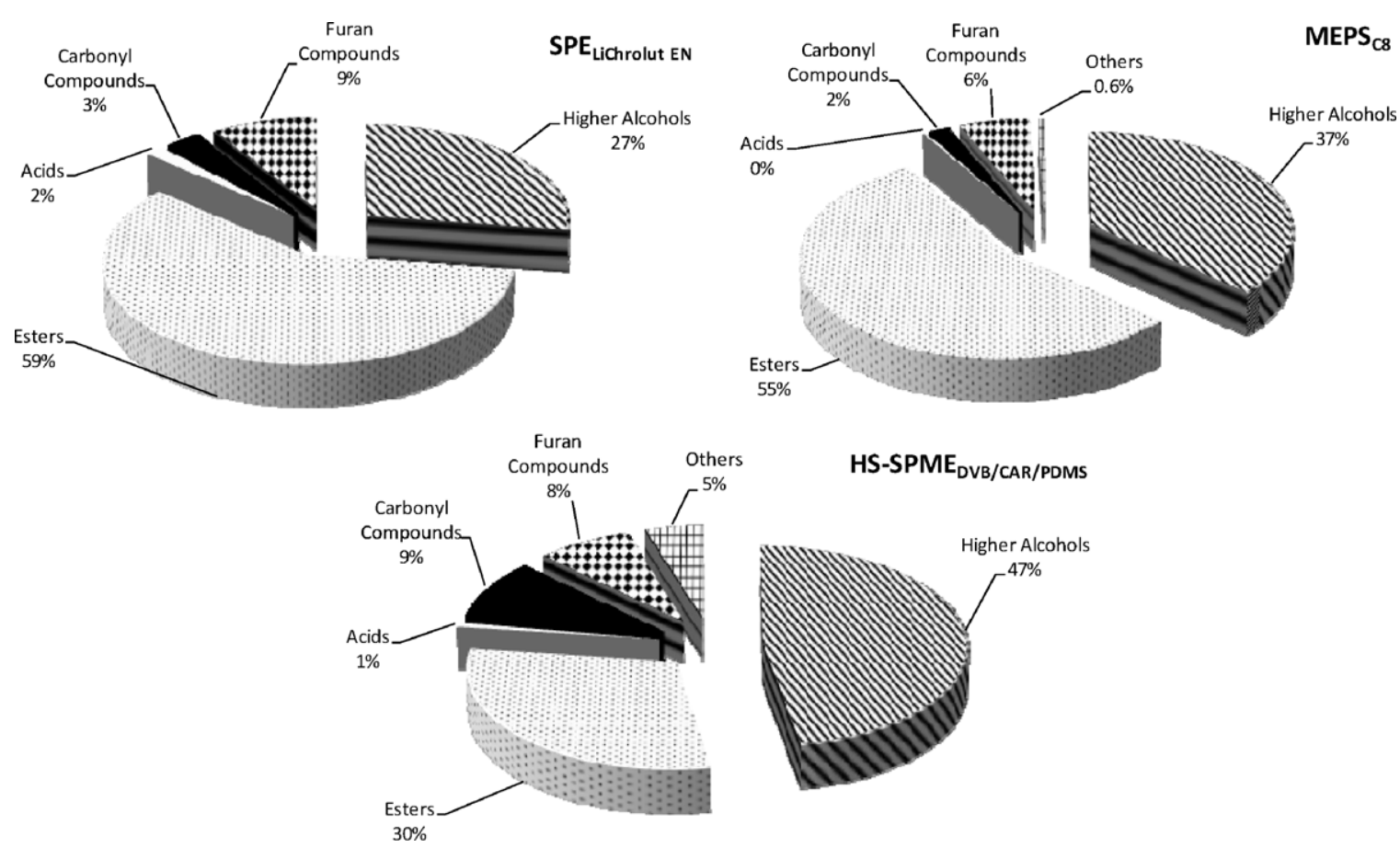

HS-SPME $E_{\text {DVB/CAR/PDMS }}$

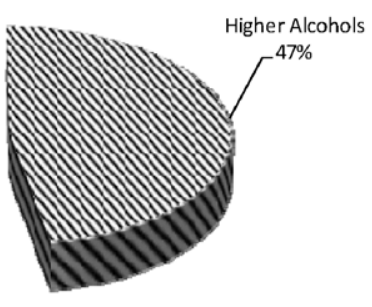

Fig. 6. Comparison of the main chemical classes of VOCs and SVOCs found in Madeira Bual wine, obtained with the best sorbents, LiChrolut EN (for SPE extraction technique); $\mathrm{C}_{8}$ (for MEPS extraction technique), and DVB/CAR/PDMS fibre (for SPME extraction technique).

obtained by using PDMS/DVB, PEG or PA fibres. CAR/PDMS fibre coating extracted $90.1 \%$ of DVB/CAR/PDMS while PA coating extracted the lowest amount (about $59.0 \%$ of DVB/CAR/PDMS). Similar amounts of volatiles (70.9-80.2\% of DVB/CAR/PDMS) were extracted using, either PDMS, PEG, or PDMS/DVB coatings (Fig. 3c). According to Fig. 3, independent of the employed fibre, higher alcohols and ethyl esters showed the most intense signals, which suggests that both chemical groups might be constituted by VOCS and SVOCs with higher concentrations.

Higher alcohols had higher affinity for $85 \mu \mathrm{m}$ PA fibre. A great affinity for CAR/PDMS and DVB/CAR/PDMS coatings was also observed for this group of compounds. These coatings also present better sensitivity for furan compounds, and carbonyl compounds. PA coating showed a strong extraction capacity for volatile fatty acids while esters are more efficiently isolated by PDMS/DVB coating (Fig. 3c). In any case, fibres containing carbon showed the best results, as obtained by other authors in the analysis of VOCs and SVOCs [59-61]. Apparently the desorption process on porous carboxen particles maximises the capture of polar compounds.

Considering the HS-SPME extraction conditions, 3-methyl-1butanol $(5 ; 36.6 \pm 1.7 \%$, on average) was the major component found in Bual wines (Table 5). The diethyl succinate (36; $18.4 \pm 3.3 \%$ ) was the second largest compound extracted with this coating, followed by 2 -phenylethanol $(\mathbf{4 4} ; 7.7 \pm 0.2 \%)$, ethyl lactate (11; $6.7 \pm 3.7 \%)$, and 2 -furfural $(21 ; 4.6 \pm 0.4 \%)$.

For 2-methyl-1-propanol (1), D-limonene (2), ethyl octanoate (17), benzaldehyde (26), 5-methyl-2-furfural (29), ethyl 2furoate (33), ethyl benzoate (35), and benzyl alcohol (41) better results were observed for DVB/CAR/PDMS, while PDMS/DVB fibre exhibited good potential to extract diethyl succinate (36), 3methyl-1-butanol (5), 2-phenylethanol (44), ethyl lactate (11), and ethyl 4-ethoxy benzoate (48). CAR/PDMS extracts with high efficiency ethyl hexanoate (6), p-cymene (9), (Z)-3-hexen-1-ol (13), 2-acetylfuran (24), whereas PDMS is more indicated to extract 1dodecene (19), decanal (22), $\alpha$-ionol (43), diethyl malate (46), and octanoic (47), tetradecanoic (55), and hexadecanoic acids (56). PA presents better results for $\gamma$-butyrolactone (31), ethyl phenylacetate (39), benzophenone (52), 5-(hydroxymethyl)-2-furfural (53) while PEG showed high extraction efficiency for ethyl lactate (45), acetic acid (20), 2,3-butanediol (28), and 2-phenylethanol (44).

\subsection{Comparison of miniaturized sorbent and solid phase microextraction techniques}

In order to compare SPE, MEPS, and SPME extraction techniques, the data obtained by analysing Bual wine samples, were compared (Tables 3-5). A total of 103 VOCs and SVOCs obtained by the three different extractive methods were classified into six different groups: (i) higher alcohols; (ii) ethyl esters; (iii) fatty acids; (iv) carbonyl compounds; (v) furan compounds; (vi) miscellaneous (Fig. 3). They were identified with an average mass spectral match of 864 with an upper and lower $95 \%$ of the mean at 923 and 815 , respectively, identified based on comparison of their mass spectra to reference database (MS), and by comparison of the RIs calculated $\left(\mathrm{RI}_{\text {calc }}\right)$ with the values reported in the literature $\left(\mathrm{RI}_{\text {lit }}\right)$ for equivalent column (Tables 3-5). An average difference ( $\left.\left|\mathrm{RI}_{\text {calc }}-\mathrm{RI}_{\text {lit }}\right|\right)$ for $\mathrm{RI}_{\text {cal }}$ compared to the $\mathrm{RI}_{\text {lit }}$ reported in the literature for similar chromatographic columns of 13.4 units with an upper and lower $95 \%$ of the mean at 84.0 (2,5-furandicarboxaldehyde) and 0 (4-ethyl phenol), respectively, was obtained. This difference in RI is considered reasonable $(<5 \%)$ if one takes into account that the literature data is obtained from a large range of GC stationary phases. Bianchi et al. [66] commented that differences in retention indices for aroma compounds on comparable stationary phases may vary between 5 and 20 units, however, larger differences have been observed [67]. Babushok et al. [68] also noted that in the development of the NIST database of retention indices, 80.427 retention indices representing 9722 species analysed on dimethylpolysiloxane stationary phases had an average deviation of 10 units but a 99th percentile deviation of 91 units [67]. The differences in calculated and reported retention indices reported in this study fall well within these values. Similar results were observed by Robinson et al. [67] on the analysis of wine 
volatiles by comprehensive two-dimensional das chromatography $(G C \times G C)$ time of flight mass spectrometry (ToFMS).

As was expected, qualitative and quantitative differences were definitely observed in the chromatographic profiles obtained by the three approaches (Figs. 1-3). Tables 3-5 display the results of the VOCs and SVOCs derived from triplicate extractions. As can be seen from the data, a total of 76 VOCs and SVOCs were isolated and identified by SPE/GC-qMS methodology, whereas only 15 and 56 compounds were positively identified by MEPS/GC-qMS and HS-SPME/GC-qMS methodologies, respectively. In addition SPE technique is able to concentrate larger amounts than MEPS and HSSPME. This suggest that SPE is generally a more sensitive technique than SPME and MEPS for the extraction of VOCs and SVOCs from wines, although in practice the SPE and SPME may complement each other quite well.

Fig. 6 compares the main chemical class of compounds identified in volatile and semi-volatile fraction of Bual wines obtained by the three techniques studied, using the best sorbents/fibre. That figure shows, without ambiguity, that the products preferentially obtained are ethyl esters for SPE and MEPS and higher alcohols for SPME.

Regarding SPE, Lichrolut-EN proved higher potential for extraction ethyl esters (59\%), furan compounds (9\%), and volatile fatty acids (2\%) than MEPS/GC-qMS and HS-SPME/GC-qMS whereas, higher alcohols (47.0\%) and carbonyl compounds (9.0\%) showed higher affinity for HS-SPMS $\mathrm{DVB}_{\mathrm{CAR} / \mathrm{PDMS}}$ than others fibre coatings and sorbents. MEPS technique using $C_{8}$ as sorbent material, exhibit high extraction capacity for esters and higher alcohols. Comparing all methods, LiChrolut EN sorbent (SPE) obtained a good response on the number of extracted compounds and total peak area. The physic-chemical processes which occurs in every extraction method, could explain the variation observed in the chemical composition, according to technique used.

The results showed that Bual wines are characterized by high peak areas, and probably high concentrations, of diethyl malate, diethyl succinate, 3-methyl-1-butanol, 2-phenylethanol and 5(hydroxymethyl)-2-furfural as determined by the three techniques utilized. The other major compounds include ethyl lactate, diethyl tartarate, ethyl citrate, and syringaldehyde.

One-way ANOVA with $p$ values $<0.05$ was achieved for the three techniques using the chemical groups constituted, higher alcohols

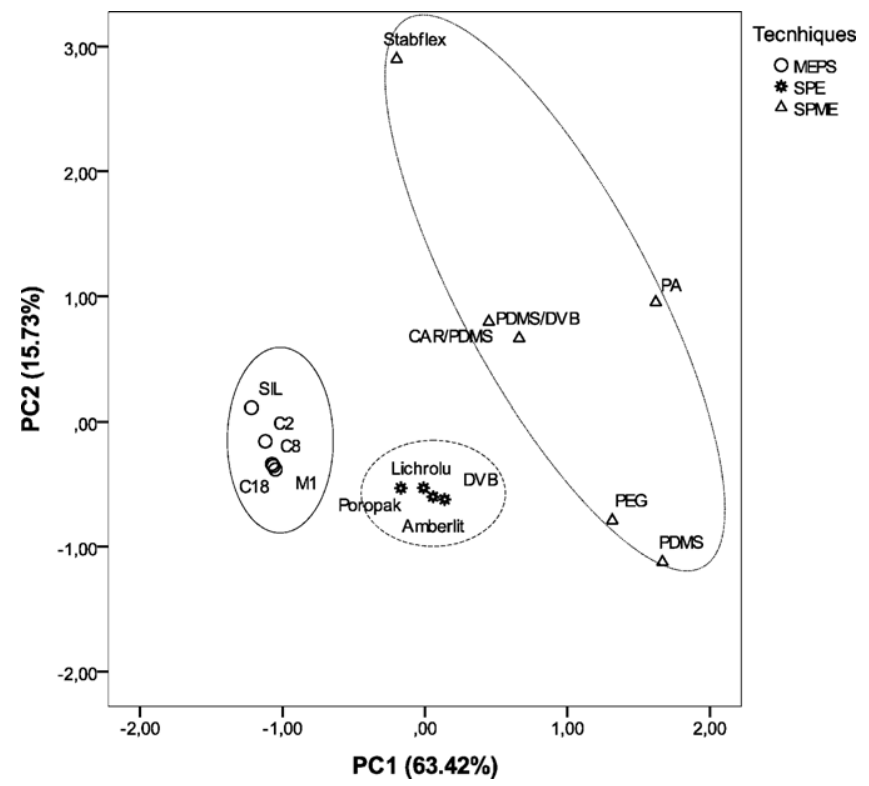

Fig. 7. Separation of SPE, SPME and MEPS extraction techniques based on PCA factor scores.
(HA) ethyl esters (EST), fatty acids (FA), carbonyl compounds (CC), furan compounds (FC) and miscellaneous compounds coded as OTHERS (SPSS, version 19.0). All chemical groups were statistically significant (ANOVA, $p<0.05$ ) between the three techniques studied (Supplementary Tables 6 and 7).

To study the principal sources of variation among the results, detect intrinsic clustering and possible outliers, and differentiate between the three techniques evaluated, exploratory PCA was applied to the GC-qMS peak areas that were obtained for the volatile and semi-volatile fraction. PCA is an unsupervised projection method that is used to visualise the dataset and display similarities and differences. PCA of the data showed that the variables selected (chemical groups) were enough to describe subsets with similar characteristics and relate to the extraction techniques. Although this set of variables only explained $79.15 \%$ of the variability between the first two PCs, it was enough to deconvolute the set of cases in three subsets according to the extraction technique (Fig. 7).

\section{Conclusions}

A total of 103 VOCs and SVOCs belonging to several chemical classes were identified in Bual Madeira wines using three different high-throughput miniaturized (ad)sorptive extraction techniques - SPE, MEPS, and SPME, and different sorbents designed to extract a broad range of analytes. The extracts resulting from distinct methodologies showed different global volatile and semi-volatile signature. Based on the extraction potential of the three methodologies and under the experimental condition employed, it can be concluded that:

i. the more traditional technique, SPE, using as sorbent the LiChrolut EN copolymer ( $\mathrm{SPE}_{\mathrm{LiChrolut} \mathrm{EN}}$ ) was the most suitable procedure for the extraction of wine VOCs and SVOCs allowing for the identification of up to 78 components, 19 and 63 more than SPME and MEPS, respectively;

ii. HS-SPME extracted more than half of VOCs and SVOCs compared with SPE technique;

iii. MEPS revealed to be the less efficient extraction technique regarding for wine VOCs and SVOCs.

Results showed that the three extraction methods studied both of them, SPE and SPME, can be used for the qualitative and quantitative determination of VOCs and SVOCs from wines. Nevertheless, when wishing to choose one of these isolation techniques, it would be convenient to consider other factors such as the amount of solvent required or the time consumption. Despite the fact that extracts obtained by means of SPE displayed a higher qualitative and quantitative composition in comparison with the other techniques tested, SPME can also be used to extract VOCs and SVOCs from wines. However, SPME is solvent-free, required smaller amount of sample and shorter extractions times. On the other hand, extraction procedure by means of MEPS was the fastest and it used minor amount of sample and solvent than SPE, nevertheless the results obtained are generally poor in comparison with SPE and SPME techniques.

\section{Acknowledgements}

The authors thanks the financial support of Interreg (Transnational Cooperation MAC 2007-2013 Program) through VinSaudeMAC Project (MAC/1/M105), and FCT through the MS Portuguese Network (REDE/1508/RNEM/2005) and Pluriannual Base Funding (QUI-Madeira-674). 


\section{Appendix A. Supplementary data}

Supplementary data associated with this article can be found, in the online version, at doi:10.1016/j.talanta.2011.10.010.

\section{References}

[1] R.B. Boulton, V.L. Singleton, L.F. Bisson, R. Kunkee, Principles and Pratices of Wine Making, Chapman and Hall, London, 1995.

[2] J.S. Câmara, M.A. Alves, J.C. Marques, Anal. Chim. Acta 555 (2006) 191-200.

[3] P. Polášková, J. Herszage, S.E. Ebeler, Chem. Soc. Rev. 37 (2008) 2478-2489.

[4] R. Perestrelo, M. Caldeira, F. Rodrigues, J.S. Camara, J. Sep. Sci. 31 (2008) $1841-1850$.

[5] R. Castro, R. Natera, P. Benitez, C.G. Barroso, Anal. Chim. Acta 513 (2004) $141-150$.

[6] M. Ortega-Heras, M.L. González-San José, S. Beltrán, Anal. Chim. Acta 458 (2002) 85-93.

[7] G.P. Blanch, G. Reglero, M. Herraiz, J. Agric. Food Chem. 43 (1995) 1251-1258.

[8] R. López, M. Aznar, J. Cacho, V. Ferreira, J. Chromatogr. A 966 (2002) 167-177.

[9] M. Isabel Spranger, M. Cristina Clímaco, B. Sun, N. Eiriz, C. Fortunato, A. Nunes, M. Conceição Leandro, M. Luísa Avelar, A. Pedro Belchior, Anal. Chim. Acta 513 (2004) 151-161.

[10] T. Hyötyläinen, Anal. Bioanal. Chem. 394 (2009) 743-758.

[11] M. Del Carlo, A. Pepe, G. Sacchetti, D. Compagnone, D. Mastrocola, A. Cichelli, Food Chem. 111 (2008) 771-777.

[12] R. Perestrelo, J.M. Nogueira, J.S. Camara, Talanta 80 (2009) 622-630.

[13] N. Loscos, P. Hernández-Orte, J. Cacho, V. Ferreira, J. Agric. Food Chem. 57 (2009) 5468-5479.

[14] M. Jesús Ibarz, V. Ferreira, P. Hernández-Orte, N. Loscos, J. Cacho, J. Chromatogr. A 1116 (2006) 217-229.

[15] V. Ferreira, R. López, A. Escudero, J.F. Cacho, J. Chromatogr. A 806 (1998) 349-354.

[16] E. Campo, J. Cacho, V. Ferreira, J. Chromatogr. A 1140 (2007) 180-188.

[17] S. Insa, E. Anticó, V. Ferreira, J. Chromatogr. A 1089 (2005) 235-242.

[18] C. Domínguez, D.A. Guillén, C.G. Barroso, Anal. Chim. Acta 458 (2002) 95102.

[19] C. Nerín, J. Salafranca, M. Aznar, R. Batlle, Anal. Bioanal. Chem. 393 (2009) 809-833.

[20] A.-R. Mohamed, J. Chromatogr. A 1217 (2010) 2569-2580.

[21] A.-R. Mohamed, J. Chromatogr. B 801 (2004) 317-321.

[22] M. Vita, P. Skansen, M. Hassan, M. Abdel-Rehim, J. Chromatogr. B 817 (2005) 303-307.

[23] M. Abdel-Rehim, P. Skansen, M. Vita, Z. Hassan, L. Blomberg, M. Hassan, Anal. Chim. Acta 539 (2005) 35-39.

[24] R. Said, Z. Hassan, M. Hassan, M. Abdel-Rehim, J. Liq. Chromatogr. Relat. Technol. 31 (2008) 683-694.

[25] A. El-Beqqali, A. Kussak, L. Blomberg, M. Abdel-Rehim, J. Liq. Chromatogr. Relat. Technol. 30 (2007) 575-586.

[26] A. El-Beqqali, A. Kussak, M. Abdel-Rehim, J. Sep. Sci. 30 (2007) 421-424.

[27] M. Abdel-Rehim, A. Andersson, A. Breitholtz-Emanuelsson, M. Sandberg-Ställ, K. Brunfelter, K.-J. Pettersson, C. Norsten-Höög, J. Chromatogr. Sci. 46 (2008) 518-523.

[28] A. Spietelun, M. Pilarczyk, A. Kloskowski, J. Namiesnik, Chem. Soc. Rev. 39 (2010) 4524-4537.

[29] J. Pawliszyn, Applications of Solid Phase Microextraction, Royal Institute of Chemistry, Cambridge, 1999.
[30] G. Ouyang, J. Pawliszyn, Anal. Chim. Acta 627 (2008) 184-197.

[31] C.L. Arthur, J. Pawliszyn, Anal. Chem. 62 (1990) 2145-2148.

[32] J. Pawliszyn, Solid Phase Microextraction Theory and Practice, Wiley-VCH Inc. New York, 1997.

[33] G. Ouyang, J. Pawliszyn, Trends Anal. Chem. 25 (2006) 692-703.

[34] L. Ferreira, R. Perestrelo, J.S. Câmara, Talanta 77 (2009) 1087-1096.

[35] M. Pontes, J.C. Marques, J.S. Câmara, Microchem. J. 93 (2009) 1-11.

[36] J. Pereira, J. Pereira, J.S. Câmara, Talanta 83 (2011) 899-906.

[37] N. Alizadeh, A. Mohammadi, M. Tabrizchi, J. Chromatogr. A 1183 (2008) 21-28.

[38] T. Kumazawa, X.-P. Lee, K. Sato, O. Suzuki, Anal. Chim. Acta 492 (2003) 49-67.

[39] F.M. Musteata, J. Pawliszyn, Trends Anal. Chem. 26 (2007) 36-45.

[40] J.L. Giraudel, L. Setkova, J. Pawliszyn, M. Montury, J. Chromatogr. A 1147 (2007) 241-253.

[41] L. Setkova, S. Risticevic, J. Pawliszyn, J. Chromatogr. A 1147 (2007) 224-240.

[42] L. Setkova, S. Risticevic, J. Pawliszyn, J. Chromatogr. A 1147 (2007) 213-223.

[43] R. Perestrelo, S. Petronilho, J.S. Câmara, S.M. Rocha,J. Chromatogr. A 1217 (2010) 3441-3445.

[44] R. Mayadunne, T.-T. Nguyen, P.J. Marriott, Anal. Bioanal. Chem. 382 (2005) 836-847.

[45] R. Perestrelo, A.S. Barros, J.S. Câmara, S.M. Rocha, J. Agric. Food Chem. 59 (2011) 3186-3204.

[46] S. Jönsson, J. Hagberg, B. van Bavel, J. Agric. Food Chem. 56 (2008) 4962-4967.

[47] H. Trutnovsky, A.B. Sakla, Anal. Chim. Acta 59 (1972) 285-291.

[48] E. Boido, A. Lloret, K. Medina, L. Fariña, F. Carrau, G. Versini, E. Dellacassa, J. Agric. Food Chem. 51 (2003) 5408-5413.

[49] C.G. Edwards, R.B. Beelman, J. Agric. Food Chem. 38 (1990) 216-220.

[50] J. Zapata, L. Mateo-Vivaracho, J. Cacho, V. Ferreira, Anal. Chim. Acta $660(2010)$ 197-205.

[51] A. Barata, E. Campo, M. Malfeito-Ferreira, V. Loureiro, J. Cacho, V. Ferreira, J. Agric. Food Chem. 59 (2011) 2543-2553.

[52] J. Ledauphin, C. Le Milbeau, D. Barillier, D. Hennequin, J. Agric. Food Chem. 58 (2010) 7782-7793.

[53] J.A. Pino, O. Queris, J. Agric. Food Chem. 59 (2011) 4885-4890.

[54] M.A. Majcher, H.H. Jelen, J. Agric. Food Chem. 59 (2011) 4932-4937.

[55] J.-K. Moon, T. Shibamoto, J. Agric. Food Chem. 58 (2010) 5465-5470.

[56] M. Aznar, R. López, J.F. Cacho, V. Ferreira, J. Agric. Food Chem. 49 (2001) 2924-2929.

[57] B. Fernández de Simón, E. Cadahía, J. Jalocha, J. Agric. Food Chem. 51 (2003) 7671-7678.

[58] K. Wada, T. Shibamoto, J. Agric. Food Chem. 45 (1997) 4362-4366.

[59] J. Bosch-Fusté, M. Riu-Aumatell, J.M. Guadayol, J. Caixach, E. López-Tamames, S. Buxaderas, Food Chem. 105 (2007) 428-435.

[60] M. Riu-Aumatell, J. Bosch-Fusté, E. López-Tamames, S. Buxaderas, Food Chem. 95 (2006) 237-242.

[61] P. Comuzzo, L. Tat, A. Tonizzo, F. Battistutta, Food Chem. 99 (2006) 217-230.

[62] V. Ferreira, R. López, M. Aznar, in: J. Jackson (Ed.), Analysis of Taste and Aroma, Springer, Berlin, 2001

[63] D. Hernanz, V. Gallo, Á.F. Recamales, A.J. Meléndez-Martínez, F.J. Heredia Talanta 76 (2008) 929-935.

[64] M.J. Gómez-Míguez, J.F. Cacho, V. Ferreira, I.M. Vicario, F.J. Heredia, Food Chem. 100 (2007) 1464-1473.

[65] J.S. Câmara, P. Herbert, J.C. Marques, M.A. Alves, Anal. Chim. Acta 513 (2004) 203-207.

[66] F. Bianchi, M. Careri, A. Mangia, M. Musci, J. Sep. Sci. 30 (2007) 563-572

[67] A.L. Robinson, P.K. Boss, H. Heymann, P.S. Solomon, R.D. Trengove, J. Chromatogr. A 1218 (2011) 504-517.

[68] V.I. Babushok, P.J. Linstrom, J.J. Reed, I.G. Zenkevich, R.L. Brown, W.G. Mallard, S.E. Stein, J. Chromatogr. A 1157 (2007) 414-421. 